\title{
Boundary central charge from bulk odd viscosity: Chiral superfluids
}

\author{
Omri Golan $\odot,{ }^{1, *}$ Carlos Hoyos, ${ }^{2}$ and Sergej Moroz ${ }^{3,4}$ \\ ${ }^{1}$ Department of Condensed Matter Physics, Weizmann Institute of Science, Rehovot 76100, Israel \\ ${ }^{2}$ Department of Physics, Universidad de Oviedo, c/ Federico Garcia Lorca 18, 33007, Oviedo, Spain \\ ${ }^{3}$ Physik-Department, Technische Universität München, D-85748 Garching, Germany \\ ${ }^{4}$ Munich Center for Quantum Science and Technology (MCQST), Schellingstr. 4, D-80799 München
}

(Received 30 March 2019; revised manuscript received 18 August 2019; published 12 September 2019)

\begin{abstract}
We derive a low-energy effective field theory for chiral superfluids, which accounts for both spontaneous symmetry breaking and fermionic ground-state topology. Using the theory, we show that the odd (or Hall) viscosity tensor, at small wave vector, contains a dependence on the chiral central charge $c$ of the boundary degrees of freedom, as well as additional nonuniversal contributions. We identify related bulk observables which allow for a bulk measurement of $c$. In Galilean invariant superfluids, only the particle current and density responses to strain and electromagnetic fields are required. To complement our results, the effective theory is benchmarked against a perturbative computation within a canonical microscopic model.
\end{abstract}

DOI: 10.1103/PhysRevB.100.104512

\section{INTRODUCTION}

The odd (or Hall) viscosity $\eta_{\mathrm{o}}$ is a nondissipative, timereversal odd, stress response to strain rate [1-5], which can appear even in superfluids (SFs) and incompressible (or gapped) fluids, where the more familiar dissipative viscosity vanishes. Observable signatures of $\eta_{\mathrm{o}}$ are actively studied in a variety of systems [6], and recently led to its measurement in a colloidal fluid [7] and in graphene [8].

In isotropic $2+1$-dimensional fluids, the odd viscosity tensor at zero wave vector $(\mathbf{q}=\mathbf{0})$ reduces to a single component. In analogy with the celebrated quantization of the odd (or Hall) conductivity in the quantum Hall $(\mathrm{QH})$ effect [9], this component obeys a quantization condition

$$
\eta_{\mathrm{o}}^{(1)}=-(\hbar / 2) s n_{0}, s \in \mathbb{Q},
$$

in incompressible quantum fluids $[1,10,11]$. Here $n_{0}$ is the ground-state density, and $s$ is a rational topological invariant labeling the many-body ground state, which corresponds to the average angular momentum per particle (in units of $\hbar$, henceforth set to 1$)$.

Remarkably, Eq. (1) also holds in certain compressible quantum fluids, which are the subject of this paper. These are chiral superfluids (CSFs), where the ground state is a condensate of Cooper pairs of fermions, which are spinning around their center of mass with an angular momentum $\ell \in$ $\mathbb{Z}$ [12-14]; see Fig. 1(a). Thin films of ${ }^{3} \mathrm{He}-\mathrm{A}$ are experimentally accessible $p$-wave $(\ell= \pm 1)$ CSFs [15], and there are proposals for the realization of various CSFs in cold atoms [16]. Closely related chiral superconductors [17] have recently been realized [18], and some of the most debated fractional QH states [19] are believed to be CSFs of composite fermions $[12,20]$. Computing $\eta_{\mathrm{o}}^{(1)}$ in an $\ell$-wave CSF, one finds Eq. (1) with the intuitive $s=\ell / 2$ [10,11,21-23]. Thus, a

*golanomri@gmail.com measurement of $\eta_{\mathrm{o}}^{(1)}$ at $\mathbf{q}=\mathbf{0}$ can be used to obtain the angular momentum of the Cooper pair, but carries no additional information.

An $\ell$-wave pairing involves the spontaneous symmetry breaking (SSB) of time reversal $T$ and parity (spatial reflection) $P$ down to $P T$, and of the symmetry groups generated by particle number $N$ and angular momentum $L$ down to a diagonal subgroup,

$$
U(1)_{N} \times S O(2)_{L} \rightarrow U(1)_{L-(\ell / 2) N},
$$

which implies a single Goldstone field, charged under the broken generator $N+(\ell / 2) L$, as well as massive Higgs fields $[24,25]$. For CSFs, it is this SSB pattern, rather than

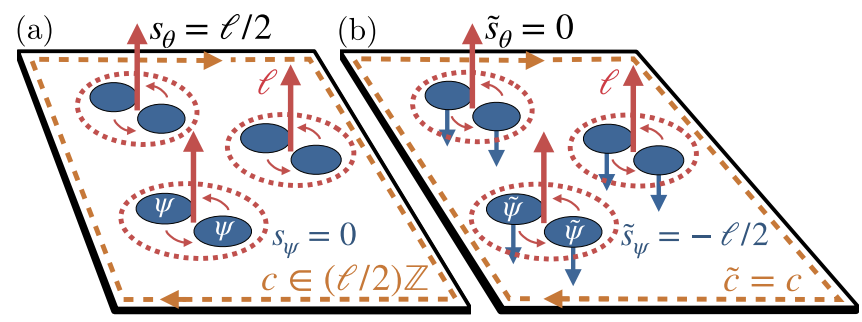

FIG. 1. (a) A CSF is composed of fermions $\psi$ which carry no geometric spin, $s_{\psi}=0$, and form Cooper pairs with a relative angular momentum $\ell \in \mathbb{Z}$ (red arrows). The geometric spin $s_{\theta}=\ell / 2$ of the Cooper pair gives rise to the $\mathbf{q}=\mathbf{0}$ odd viscosity (1), with $s=s_{\theta}$. The CSF supports boundary degrees of freedom (dashed orange) with a chiral central charge $c \in(\ell / 2) \mathbb{Z}$, which cannot be extracted from the odd viscosity $\eta_{\mathrm{o}}(\mathbf{q})$ alone Eq. (18). (b) In an auxiliary CSF, the fermion $\tilde{\psi}$ is assigned a geometric spin $\tilde{s}_{\psi}=-\ell / 2$ (blue arrows). The geometric spin of the Cooper pair therefore vanishes, $\tilde{s}_{\theta}=\ell / 2+\tilde{s}_{\psi}=0$, as in an $s$-wave superfluid, but the central charge is unchanged, $\tilde{c}=c$. As a result, the small $\mathbf{q}$ behavior of the odd viscosity $\tilde{\eta}_{\mathrm{o}}$ depends only on $c$ Eq. (19). The improved odd viscosity of the CSF is defined as the odd viscosity of the auxiliary CSF, and is given explicitly by Eq. (22). 
ground-state topology, which implies the quantization $s=$ $\ell / 2[21,26]$.

Nevertheless, a CSF with fixed $\ell$ does have a nontrivial ground-state topology-single fermion excitations are gapped, and the fermionic ground state can be assigned a topological invariant. This is the boundary chiral central charge $c \in(\ell / 2) \mathbb{Z}$ (per spin component) [12,13], which counts the net chirality of $1+1$ dimensional Majorana spinors present on the boundary between the CSF and vacuum. For example, a $p$-wave CSF composed of spin-less fermions has a minimal nonvanishing $c= \pm 1 / 2$, while a $d$-wave $(\ell= \pm 2)$ CSF, which requires spin-full fermions, has a minimal nonvanishing $c= \pm 2$, or $c= \pm 1$ per spin component.

The invariant $c$ determines the boundary gravitational anomaly [27], and the boundary thermal Hall conductance $[12,28,29]$, which has been measured in recent experiments on $\mathrm{QH}$ and spin systems [30]. Based on the fundamental principle of anomaly inflow [31-34] it is expected that $c$ can be measured in the bulk of a CSF, but whether this is indeed the case, and if so, what should actually be measured, has so far remained unclear. Providing an answer to this question is the main goal of the present paper.

Analysis of the problem has previously been carried out only within the relativistic limit of the $p$-wave CSF, where the nonrelativistic kinetic energy of the fermions is neglected [12,25,32,35-37]. Within this limit one finds a bulk gravitational Chern-Simons (gCS) term, which implies a $c$-dependent correction to $\eta_{\mathrm{o}}^{(1)}$ of (1) at small nonzero wave vector [37-39],

$$
\delta \eta_{\mathrm{o}}^{(1)}(\mathbf{q})=-\frac{c}{24} \frac{1}{4 \pi} q^{2} .
$$

One is therefore led to suspect that $c$ can be obtained from the $q^{2}$ correction to $\eta_{\mathrm{o}}$, but the fate of this correction beyond the relativistic limit remains unclear.

In particular, the relativistic limit misses most of the physics of the Goldstone field [25]. Analysis of the Goldstone physics in CSFs was undertaken in Refs. [40,41]. More recently, Refs. [21,23] considered CSFs in curved (or strained) space, following the pioneering work [42] on $s$-wave $(\ell=$ $0)$ SFs. These works demonstrated that the Goldstone field, owing to its charge $L+(\ell / 2) N$, produces the $\mathbf{q}=\mathbf{0}$ odd viscosity Eq. (1), and it is therefore natural to expect that a $q^{2}$ correction similar to Eq. (3) will also be produced. Nevertheless, Refs. [21,23] did not consider the derivative expansion to the high order at which $q^{2}$ corrections to $\eta_{\mathrm{o}}$ would appear, nor did they detect any bulk signature of $c$ at lower orders.

In this paper we obtain a low-energy effective field theory that captures both SSB and fermionic ground-state topology, which extends and unifies the aforementioned results of $[21,23,42]$ and $[12,25,32,35-37]$. Using the theory we compute the $q^{2}$ correction to $\eta_{\mathrm{o}}$, and provide several routes towards the bulk measurement of the boundary central charge in CSFs.

We note that there is an ongoing discussion in the literature regarding a possible bulk thermal Hall conductivity proportional to $c$, including some contradicting results $[43,44]$. This provides further motivation to study the appearance of $c$ in the bulk odd viscosity.

\section{BUILDING BLOCKS OF THE EFFECTIVE FIELD THEORY}

To probe a CSF, we minimally couple it to two background fields - a time-dependent spatial metric $G_{i j}$, which we use to apply strain $u_{i j}=\left(G_{i j}-\delta_{i j}\right) / 2$ and strain rate $\partial_{t} u_{i j}$, and a $U(1)_{N}$-gauge field $A_{\mu}=\left(A_{t}, A_{i}\right)$, where we absorb a chemical potential $A_{t}=-\mu+\cdots$. The microscopic action $S$ is then invariant under $U(1)_{N}$ gauge transformations, implying the number conservation $\partial_{\mu}\left(\sqrt{G} J^{\mu}\right)=0$, where $\sqrt{G} J^{\mu}=$ $-\delta S / \delta A_{\mu}$. It is also clear that $S$ is invariant under spatial diffeomorphisms generated by $\delta x^{i}=\xi^{i}(\mathbf{x})$, if $G_{i j}$ transforms as a tensor and $A_{\mu}$ as a 1-form. Less obvious is the fact that a Galilean invariant fluid is additionally symmetric under $\delta x^{i}=\xi^{i}(t, \mathbf{x})$, provided one adds to the transformation rule of $A_{i}$ a nonstandard mass-dependent piece [21,42,45-50],

$$
\delta A_{i}=-\xi^{k} \partial_{k} A_{i}-A_{k} \partial_{i} \xi^{k}+m G_{i j} \partial_{t} \xi^{j} .
$$

We refer to $\delta x^{i}=\xi^{i}(\mathbf{x}, t)$ as local Galilean symmetry (LGS), as it can be viewed as a local version of the Galilean transformation $\delta x^{i}=v^{i} t$. The LGS implies the momentum conservation law

$$
\frac{1}{\sqrt{G}} \partial_{t}\left(\sqrt{G} m J^{i}\right)+\nabla_{j} T^{j i}=n E_{i}+\varepsilon^{i j} J_{j} B,
$$

where $\sqrt{G} T^{i j}=2 \delta S / \delta G^{i j}$ is the stress tensor and the right hand side is the Lorentz force. This fixes the momentum density $P^{i}=m J^{i}$ - a familiar Galilean relation.

Since CSFs spontaneously break the rotation symmetry in flat space, to describe them in curved, or strained, space, it is necessary to introduce a background vielbein. This is a field $E_{j}^{A}$ valued in $G L(2)$, such that $G_{i j}=E_{i}^{A} \delta_{A B} E_{j}^{B}$, where $A, B \in\{1,2\}$. For a given metric $G$ the vielbein $E$ is not unique-there an internal $O(2)_{P, L}=\mathbb{Z}_{2, P} \ltimes S O(2)_{L}$ ambiguity, or symmetry, acting by $E_{j}^{A} \mapsto O_{B}^{A} E_{j}^{B}, O \in O(2)_{P, L}$. The generators $L, P$ correspond to internal spatial rotations and reflections, and are analogs of angular momentum and spatial reflection (parity) on the tangent space. The inverse vielbein $E_{B}^{j}$ is defined by $E_{j}^{A} E_{B}^{j}=\delta_{B}^{A}$.

The charge $N+(\ell / 2) L$ of the Goldstone field $\theta$ implies the covariant derivative

$$
\nabla_{\mu} \theta=\partial_{\mu} \theta-A_{\mu}-s_{\theta} \omega_{\mu},
$$

with a geometric spin $s_{\theta}=\ell / 2$. Here $\omega_{\mu}$ is the nonrelativistic spin connection, an $S O(2)_{L}$ gauge field which is $E_{j}^{A}$ compatible; see Appendix A. So far we assumed that the microscopic fermion $\psi$ does not carry a geometric spin, $s_{\psi}=0$, which defines the physical system of interest. It will be useful, however, to generalize to $s_{\psi} \in(1 / 2) \mathbb{Z}$, where the covariant derivative of the fermion is

$$
\nabla_{\mu} \psi=\left(\partial_{\mu}+i A_{\mu}+i s_{\psi} \omega_{\mu}\right) \psi .
$$

A nonzero $s_{\psi}$ modifies the geometric spin of $\theta$ to $s_{\theta}=s_{\psi}+$ $\ell / 2$, and the unbroken generator in Eq. (2) to $L-s_{\theta} N$. In the special case $s_{\psi}=-\ell / 2$ the Cooper pair is geometrically spin-less and $L$ is unbroken, as in an $s$-wave SF; see Fig. 1(b). This $s_{\theta}=0 \mathrm{CSF}$ is, however, distinct from a conventional $s$-wave SF, because $P$ and $T$ are still broken down to $P T$, and we therefore refer to it as a geometric $s$-wave ( $g s$-wave) CSF, to distinguish the two. In particular, a central charge $c \neq 0$, 
which is $P, T$-odd, is not forbidden, and is in fact independent of $s_{\psi}$. This makes the gs-wave CSF particularly useful for our purposes.

We note that $\omega_{\mu}$ transforms as a 1-form under LGS only if $B / 2 m$ is added to $\omega_{t}$ [21,23], which we do implicitly throughout the paper. For $\psi$, this is equivalent to adding a g-factor $g_{\psi}=2 s_{\psi}[48]$.

\section{EFFECTIVE FIELD THEORY}

Based on the above characterization of CSFs, the lowenergy, long-wavelength behavior of the system can be captured by an effective action $S_{\text {eff }}[\theta ; A, G]$, obtained by integrating out all massive degrees of freedom-the single fermion excitations and the Higgs fields. In this section we describe a general expression for $S_{\text {eff }}$, compatible with the symmetries, SSB pattern, and ground-state topology of CSFs.

The effective action can be written order by order in a derivative expansion, with the power counting scheme [21,42]

$$
\partial_{\mu}=O(p), \quad A_{\mu}, G_{i j}=O(1), \quad \theta=O\left(p^{-1}\right) .
$$

The spin connection is a functional of $G_{i j}$ that involves a single derivative [see Eq. (A2)], so $\omega_{\mu}=O(p)$. Denoting by $\mathcal{L}_{n}$ the term in the Lagrangian which is $O\left(p^{n}\right)$ and invariant under all symmetries, we have $S_{\text {eff }}=\sum_{n=0}^{\infty} \int d^{2} x d t \sqrt{G} \mathcal{L}_{n}$. The desired $q^{2}$ corrections to $\eta_{\mathrm{o}}$ are $O\left(p^{3}\right)$, which poses the main technical difficulty.

The leading-order Lagrangian

$$
\mathcal{L}_{0}=P(X), \quad X=\nabla_{t} \theta-\frac{1}{2 m} G^{i j} \nabla_{i} \theta \nabla_{j} \theta,
$$

was studied in Ref. [21] and contains the earlier results of Ref. [40]. Here $X$ is the unique $O(1)$ scalar, which reduces to the chemical potential $\mu$ in the ground state(s) $\partial_{\mu} \theta=0$, and $P$ is an arbitrary function of $X$ that physically corresponds to the ground-state pressure $P_{0}=P(\mu)$. The function $P$ also determines the ground-state density $n_{0}=P^{\prime}(\mu)$, and the leading dispersion of the Goldstone mode $\omega^{2}=c_{s}^{2} q^{2}$, where $c_{s}^{2}=$ $\partial_{n_{0}} P_{0} / m=P^{\prime} / P^{\prime \prime} m$ is the speed of sound, squared. For $\ell \neq 0$, the spin connection appears in each $\nabla \theta$ Eq. (6), and so $\mathcal{L}_{0}$ includes $O(p)$ contributions, which produce the leading odd viscosity and conductivity, discussed below. There are no additional terms at $O(p)$, so that $\mathcal{L}_{1}=0$ [21].

At $O\left(p^{2}\right)$ one has

$$
\begin{aligned}
\mathcal{L}_{2}= & F_{1}(X) R+F_{2}(X)\left[m K_{i}^{i}-\nabla^{2} \theta\right]^{2} \\
& +F_{3}(X)\left[2 m\left(\nabla_{i} K_{j}^{j}-\nabla^{j} K_{j i}\right) \nabla^{i} \theta\right]+\cdots,
\end{aligned}
$$

where $K_{i j}=\partial_{t} G_{i j} / 2$ and $R$ are the extrinsic curvature and Ricci scalar of the spatial slice at time $t$ [51], the $F$ s are arbitrary functions of $X$, and dots indicate additional terms which do not contribute to $\eta_{\mathrm{o}}$ up to $O\left(p^{2}\right)$; see Appendix C 2 for the full expression. The Lagrangian $\mathcal{L}_{2}$ was obtained in Ref. [42] for $s$-wave SFs. For $\ell \neq 0$ the spin connection in $\nabla \theta$ produces $O\left(p^{3}\right)$ contributions to $\mathcal{L}_{2}$, and, in turn, nonuniversal $q^{2}$ corrections to $\eta_{\mathrm{o}}$.

The term $\mathcal{L}_{3}$ is the last ingredient required for reliable results at $O\left(p^{3}\right)$. Most importantly, it includes the (nonrela- tivistic) gCS term [31,33,37,52-56]

$$
\mathcal{L}_{3} \supset \mathcal{L}_{\mathrm{gCS}}=-\frac{c}{48 \pi} \omega d \omega,
$$

where the $c$-dependence is required to match the boundary gravitational anomaly [25,31,33], and $\omega d \omega=\varepsilon^{\mu \nu \rho} \omega_{\mu} \partial_{\nu} \omega_{\rho}$. Unlike the lower-order terms, $\mathcal{L}_{\mathrm{gCS}}$ is independent of $\theta$, and encodes only the response of the gapped fermions to the background fields. In Appendices B 5 and C 4 we argue that additional terms in $\mathcal{L}_{3}$ do not produce $q^{2}$ corrections to $\eta_{\mathrm{o}}$.

There are three topological terms that can be added to $S_{\text {eff }}$ [37-39,46,57-63]. These are the $U(1)$ Chern-Simons (CS) and first and second Wen-Zee (WZ1, WZ2) terms, which can be added to $\mathcal{L}_{1}, \mathcal{L}_{2}, \mathcal{L}_{3}$, respectively [64],

$$
\frac{v}{4 \pi}\left(A d A-2 \bar{s} \omega d A+\overline{s^{2}} \omega d \omega\right) .
$$

As our notation suggests, WZ2 and gCS are identical for the purpose of local bulk responses, of interest here, but the two are globally distinct $[37,56,63]$. Based on symmetry, and ignoring boundary physics, the independent coefficients $v, v \bar{s}, v \overline{s^{2}}$ obey certain quantization conditions [54] but are otherwise unconstrained. The absence of a boundary $U(1)_{N^{-}}$ anomaly then fixes $v=0$ [25], but leaves $v \bar{s}, v \overline{s^{2}}$ undetermined $[37,56,63]$. One can argue that a Chern-Simons term can only appear for the unbroken generator $L-s_{\theta} N$, so that $v=0$ implies $v \bar{s}=v \overline{s^{2}}=0$. Moreover, in the following section we will see that a perturbative computation within a canonical model for $\ell= \pm 1$ shows that $v \bar{s}=v \overline{s^{2}}=0$, which applies to any deformation of the model (which preserves the symmetries, SSB pattern, and single fermion gap), due to the quantization of $v \bar{s}, v \overline{s^{2}}$. Accordingly, we set $v \bar{s}=v \overline{s^{2}}=0$ in the following.

\section{BENCHMARKING THE EFFECTIVE THEORY AGAINST A MICROSCOPIC MODEL}

In this section we take a complementary approach and compute $S_{\text {eff }}$ perturbatively, starting from a canonical microscopic model for a spinless $p$-wave CSF. The perturbative computation verifies the general expression in a particular example, and determines the coefficients of topological terms which are not completely fixed by symmetry. It also gives one a sense of the behavior of the coefficients of nontopological terms as a function of microscopic parameters. Here we will outline the computation and describe its results, deferring many technical details to Appendix E.

The microscopic model is given by

$$
\begin{aligned}
S_{\mathrm{m}}= & \int d^{2} x d t \sqrt{G}\left[\frac{i}{2} \psi^{\dagger} \overleftrightarrow{\nabla_{t}} \psi-\frac{1}{2 m} G^{i j} \nabla_{i} \psi^{\dagger} \nabla_{j} \psi\right. \\
& \left.+\left(\frac{1}{2} \Delta^{j} \psi^{\dagger} \nabla_{j} \psi^{\dagger}+\text { h.c }\right)-\frac{1}{2 \lambda} G_{i j} \Delta^{i *} \Delta^{j}\right],
\end{aligned}
$$

where $\nabla_{\mu} \psi=\left(\partial_{\mu}+i A_{\mu}\right) \psi$, so $s_{\psi}=0$. Apart from the standard nonrelativistic kinetic term, the action includes the simplest attractive two-body interaction $[65,66]$, mediated by the complex vector $\Delta^{i}$, the order parameter, with coupling constant $\lambda>0$. 
For a given $\Delta^{j}$, the fermion $\psi$ is gapped, unless the chemical potential $\mu$ or chirality $\ell=\operatorname{sgn}\left[\operatorname{Im}\left(\Delta^{x} \Delta^{y *}\right)\right]$ are tuned to 0 , and forms a fermionic topological phase characterized by the boundary chiral central charge $[12,13,67]$

$$
c=-(\ell / 2) \Theta(\mu) \in\{0, \pm 1 / 2\} .
$$

An effective action $S_{\text {eff, }}[\Delta ; A, G]$ for $\Delta^{j}$ in the background $A_{\mu}, G_{i j}$ is then obtained by integrating over the fermion. The subscript " $\mathrm{m}$ " indicates that this is obtained from the particular microscopic model $S_{\mathrm{m}}$. Since Eq. (13) is quadratic in $\psi, \psi^{\dagger}$, obtaining $S_{\text {eff,m }}$ is formally straightforward, and leads to a functional Pfaffian.

To zeroth order in derivatives, the action $S_{\text {eff,m }}$ is given by a potential for $\Delta^{i}$, which is minimized by the $p_{x} \pm i p_{y}$ configurations. In flat space these are given by the familiar $\Delta^{j} \partial_{j}=\Delta_{0} e^{-2 i \theta}\left(\partial_{x} \pm i \partial_{y}\right)$. Here $\Delta_{0}$ is a fixed function of $m, \mu$ and $\lambda$, determined by the minimization, while the phase $\theta$ and chirality $\ell= \pm 1$ are undetermined. To write down the $p_{x} \pm i p_{y}$ configurations in curved space it is necessary to use a background vielbein $[12,21,23,66,68]$,

$$
\Delta^{j}=\Delta_{0} e^{-2 i \theta}\left(E_{1}^{j} \pm i E_{2}^{j}\right) .
$$

Fluctuations of $\Delta$ away from these configurations correspond to massive Higgs modes, which should in principle be integrated out to obtain a low-energy action $S_{\text {eff, }}[\theta ; A, G]$ that can be compared with the general $S_{\text {eff }}$ of the previous section. We will simply ignore these fluctuations, and obtain $S_{\text {eff, } \mathrm{m}}[\theta ; A, G]$ by plugging Eq. (15) into $S_{\text {eff, }}[\Delta ; A, G]$. This will suffice as a derivation of $S_{\text {eff }}$ from a microscopic model. A proper treatment of the massive Higgs modes will only further renormalize the coefficients we find, apart from the central charge $c$.

To practically compare the actions $S_{\text {eff }}$ and $S_{\text {eff,m }}$ we expand them in fields, to second order around $\theta=0, A_{\nu}=$ $-\mu \delta_{v}^{t}, G_{i j}=\delta_{i j}$, and in derivatives, to third order; see Appendices $\mathrm{C}$ and $\mathrm{E}$. Equating these two double expansions leads to an overdetermined system of equations for the phenomenological parameters in $S_{\text {eff }}$ in terms of the microscopic parameters in $S_{\mathrm{m}}$, with a unique solution. In particular, we find the dimensionless parameters

$$
\begin{aligned}
\frac{P^{\prime \prime}}{m} & =\frac{1}{2 \pi}\left\{\begin{array}{l}
1 \\
\frac{1}{1+2 \kappa}
\end{array}, \quad F_{1}^{\prime}=\frac{1}{96 \pi}\left\{\begin{array}{l}
1 \\
\frac{3}{1+2 \kappa}
\end{array},\right.\right. \\
m F_{2} & =-\frac{1}{128 \pi}\left\{\begin{array}{l}
1+2 \kappa \\
\frac{1}{1+2 \kappa}
\end{array}, \quad m F_{3}=\frac{1}{48 \pi}\left\{\begin{array}{l}
1+\kappa \\
\frac{1}{1+2 \kappa}
\end{array},\right.\right. \\
c & =\left\{\begin{array}{l}
-\ell / 2 \\
0
\end{array},\right.
\end{aligned}
$$

where $\kappa=|\mu| / m \Delta_{0}^{2}>0$, and the upper and lower values refer to $\mu>0$ and $\mu<0$, respectively. We note that for $\mu>0$ there is a single particle Fermi surface with energy $\varepsilon_{F}=\mu$ and wave vector $k_{F}=\sqrt{2 m \mu}$, which for small $\lambda$ will acquire an energy gap $\varepsilon_{\Delta}=\Delta_{0} k_{F} \ll \varepsilon_{F}$. In this weakcoupling regime, it is natural to parametrize the coefficients in Eq. (16) using the small parameter $\varepsilon_{\Delta} / \varepsilon_{F}=\sqrt{2 / \kappa}$.

The coefficient $P^{\prime \prime}$ determines the leading odd (or Hall) conductivity and has been computed previously in the literature [40,41], while $F_{1}, F_{2}$ and $F_{3}$, to the best of our knowledge, have not been computed previously, even for an $s$-wave SF.
Crucially, Eq. (16) shows that the coefficient $c$ of the bulk gCS term Eq. (11) matches the known boundary central charge Eq. (14). It follows that there is no WZ2 term in $S_{\text {eff, } \mathrm{m}}$, so $\nu \overline{s^{2}}=0$, in accordance with the previous section. We additionally confirm that $v=v \bar{s}=0$. The direct confirmation of the gCS term and its coefficient within a nonrelativistic microscopic model has been anticipated for some time $[12,25,32,35,36]$, and is the main result of the perturbative computation.

A few additional comments regarding Eq. (16) are in order:

(1) The seeming quantization of $P^{\prime \prime} / m$ and $F_{1}^{\prime}$ for $\mu>0$ is a nongeneric result, as was shown explicitly for $P^{\prime \prime} / m$ [41].

(2) The free fermion limit $\kappa \rightarrow \infty$, or $\Delta_{0} \rightarrow 0$, of certain coefficients in Eq. (16) diverges for $\mu>0$ but not for $\mu<0$. This signals the breakdown of the gradient expansion for a gapless Fermi surface, but not for gapped free fermions.

(3) The opposite limit, $\kappa \rightarrow 0$, or $m \rightarrow \infty$, is the relativistic limit mentioned above, in which the fermionic part of the model reduces to a $2+1$-dimensional Majorana spinor with mass $\mu$ and speed of light $\Delta_{0}$, coupled to Riemann-Cartan geometry described by $\Delta^{i}, A_{\mu}$, and in which $S_{\text {eff,m }}$ was already computed [25,69]. Accordingly, the limit $\kappa \rightarrow 0$ of Eq. (16) indeed reproduces the results of Refs. $[25,69]$ in a suitable sense; see Appendix E.

\section{INDUCED ACTION AND LINEAR RESPONSE}

Having derived and benchmarked the effective theory, we are now in a position to obtain linear response functions, in particular the $q^{2}$ corrections to the odd viscosity, and related observables that allow for the bulk measurement of $c$.

By expanding $S_{\text {eff }}$ to second order in the fields $\theta, A_{t}-$ $\mu, A_{i}, u_{i j}$, and performing Gaussian integration over $\theta$, we obtain an induced action $S_{\text {ind }}\left[A_{\mu}, u_{i j}\right]$ that captures the linear response of CSFs to the background fields; see Appendix D for explicit expressions. Taking functional derivatives, one obtains the expectation values $J^{\mu}=$ $G^{-1 / 2} \delta S_{\text {ind }} / \delta A_{\mu}, T^{i j}=G^{-1 / 2} \delta S_{\text {ind }} / \delta u_{i j}$ of the current and stress, and from them the conductivity $\sigma^{i j}=\delta J^{i} / \delta E_{j}$, the viscosity $\eta^{i j, k l}=\delta T^{i j} / \delta \partial_{t} u_{k l}$, and the mixed response function $\kappa^{i j, k}=\delta T^{i j} / \delta E_{k}=\delta J^{k} / \delta \partial_{t} u_{i j}$. We will also need the static susceptibilities $\chi_{J J}^{\mu, \nu}, \chi_{T J}^{i j, v}$, defined by restricting to time independent $A_{\mu}, u_{i j}$, and computing $\delta J^{\mu} / \delta A_{v}$ and $\delta J^{\nu} / \delta u_{i j}$, respectively.

Before computing $\eta_{\mathrm{o}}$, it is useful to restrict its form based on dimensionality and symmetries: space-time translations, spatial rotations, and $P T$. The analysis is performed in Appendices B 1-B 4 and results in the expression

$$
\eta_{\mathrm{o}}(\omega, \mathbf{q})=\eta_{\mathrm{o}}^{(1)} \sigma^{x z}+\eta_{\mathrm{o}}^{(2)}\left[\left(q_{x}^{2}-q_{y}^{2}\right) \sigma^{0 x}-2 q_{x} q_{y} \sigma^{0 z}\right],
$$

written in the basis $\sigma^{a b}=2 \sigma^{[a} \otimes \sigma^{b]}$ of antisymmetrized tensor products of the symmetric Pauli matrices [2]. As components of the strain tensor, the matrices $\sigma^{x}, \sigma^{z}$ correspond to shears, while the identity matrix $\sigma^{0}$ corresponds to a dilatation. The details of the system are encoded in two independent coefficients $\eta_{\mathrm{o}}^{(1)}, \eta_{\mathrm{o}}^{(2)} \in \mathbb{C}$, which are functions of $\omega, q^{2}$. At $\mathbf{q}=\mathbf{0}$ the odd viscosity tensor reduces to a single component, $\eta_{\mathrm{o}}(\omega, \mathbf{0})=\eta_{\mathrm{o}}^{(1)}(\omega) \sigma^{x z}$, as is well known [1-5]. 
The additional component $\eta_{\mathrm{o}}^{(2)}$ has not been discussed much in the literature [38,70], and also appears in the presence of (pseudo)vector anisotropy [71,72], in which case $\mathbf{q}$ should be replaced by a background (pseudo)vector b. Equation (17) applies at finite temperature, out of equilibrium, and in the presence of disorder that preserves the symmetries on average. For clean systems at zero temperature, $\eta_{\mathrm{o}}^{(1)}, \eta_{\mathrm{o}}^{(2)}$ are both real, even functions of $\omega$. In gapped systems $\eta_{\mathrm{o}}^{(1)}, \eta_{\mathrm{o}}^{(2)}$ will usually be regular at $\omega=0=q^{2}$, though exceptions to this rule have recently been found [73].

For the CSF, we find the $\omega=0$ coefficients

$$
\begin{aligned}
& \eta_{\mathrm{o}}^{(1)}\left(q^{2}\right)=-\frac{1}{2} s_{\theta} n_{0}-\left(\frac{c}{24} \frac{1}{4 \pi}+s_{\theta} C^{(1)}\right) q^{2}+O\left(q^{4}\right), \\
& \eta_{\mathrm{o}}^{(2)}\left(q^{2}\right)=\frac{1}{2} s_{\theta} n_{0} q^{-2}+\left(\frac{c}{24} \frac{1}{4 \pi}+s_{\theta} C^{(2)}\right)+O\left(q^{2}\right),
\end{aligned}
$$

where $C^{(1)}, C^{(2)} \in \mathbb{R}$ are generically nonzero, and are given by particular linear combinations of the dimensionless coefficients $F_{1}^{\prime}(\mu), m F_{2}(\mu)$, and $m F_{3}(\mu)$ defined in Eq. (10); see Appendix D for more details.

The leading term in $\eta_{\mathrm{o}}^{(1)}$ is the familiar Eq. (1), which also appears in gapped states, while the nonanalytic leading term in $\eta_{\mathrm{o}}^{(2)}$ occurs because the superfluid is gapless, and does not appear when $q \rightarrow 0$ at $\omega \neq 0$ [21]. Both leading terms obey the same quantization condition due to SSB, and are independent of $c$. The subleading corrections to both $\eta_{\mathrm{o}}^{(1)}, \eta_{\mathrm{o}}^{(2)}$ contain the quantized gCS contributions proportional to $c$, but also the nonuniversal coefficients $C^{(1)}, C^{(2)}$. Thus, $c$ cannot be extracted from a measurement of $\eta_{\mathrm{o}}$ alone.

Noting that the nonuniversal subleading corrections to $\eta_{\mathrm{o}}$ originate from the geometric spin $s_{\theta}=\ell / 2$ of the Goldstone field, one is naturally led to consider the $\mathrm{g} s$-wave CSF, where $s_{\theta}=0$ and the odd viscosity is, to leading order in $q$, purely due to $\mathcal{L}_{\mathrm{gCS}}$,

$$
\begin{aligned}
& \tilde{\eta}_{\mathrm{o}}^{(1)}\left(q^{2}\right)=-\frac{c}{24} \frac{1}{4 \pi} q^{2}+O\left(q^{4}\right), \\
& \tilde{\eta}_{\mathrm{o}}^{(2)}\left(q^{2}\right)=\frac{c}{24} \frac{1}{4 \pi}+O\left(q^{2}\right) .
\end{aligned}
$$

Here and below we use $O$ and $\tilde{O}$, for the quantity $O$ in the CSF and in the corresponding gs-wave CSF, respectively. Equation (19) follows from Eq. (18) by setting $s_{\theta}=0$, but can be

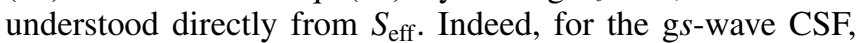
$S_{\text {eff }}$ is identical to that of the conventional $s$-wave SF to $O\left(p^{2}\right)$ but contains the additional $\mathcal{L}_{\mathrm{gCS}}$ at $O\left(p^{3}\right)$, which produces Eq. (19).

Due to the LGS Eqs. (4) and (5), the viscosity Eq. (19) implies also

$$
\tilde{\chi}_{T J, \mathrm{o}}^{i j, k}=-\frac{i}{m} \frac{c}{48 \pi} q_{\perp}^{i} q_{\perp}^{j} q_{\perp}^{k}+O\left(q^{4}\right),
$$

where $q_{\perp}^{i}=\varepsilon^{i j} q_{j}$, and the subscript "o" ("e") refers to the $P, T$-odd (even) part of an object. Thus, a steady $P, T$-odd current $\tilde{J}_{\mathrm{o}}^{k}=-\frac{1}{m} \frac{c}{96 \pi} \partial_{\perp}^{k} R+O\left(q^{4}\right)$ flows perpendicularly to gradients of curvature $R=-2 \partial_{\perp}^{i} \partial_{\perp}^{j} u_{i j}$. We conclude that, in the gs-wave CSF, $c$ can be extracted from a measurement of $\tilde{\eta}_{\mathrm{o}}$, and in the Galilean invariant case, also from a measurement of the current $\tilde{J}$ in response to strain.
Though the simple results above do not apply to the physical system of interest, the CSF, there is a relation between the observables of the CSF and the corresponding gs-wave CSF, which we can utilize. At the level of induced actions, it is given by

$$
\tilde{S}_{\text {ind }}\left[A_{\mu}, u_{i j}\right]=S_{\text {ind }}\left[A_{\mu}-(\ell / 2) \omega_{\mu}, u_{i j}\right],
$$

where $\omega_{\mu}$ is expressed through $u_{i j}$ as in Appendix A, and by taking functional derivatives one obtains relations between response functions [48]. In particular,

$$
\begin{aligned}
\tilde{\eta}_{\mathrm{o}}^{i j, k l}= & \eta_{\mathrm{o}}^{i j, k l}-\frac{\ell}{4} n_{0}\left(\sigma^{x z}\right)^{i j, k l} \\
& +\frac{i \ell}{4}\left(\kappa_{\mathrm{e}}^{i j,(k} q_{\perp}^{l)}-\kappa_{\mathrm{e}}^{k l,(i} q_{\perp}^{j)}\right)+\frac{\ell^{2}}{16} \sigma_{\mathrm{o}} q_{\perp}^{(i} \varepsilon^{j)(k} q_{\perp}^{l)},
\end{aligned}
$$

where the response functions $\eta_{\mathrm{o}}, \sigma_{\mathrm{o}}, \kappa_{\mathrm{e}}$ depend on $\omega$, q. In a Galilean invariant system one further has

$$
\begin{aligned}
\tilde{\chi}_{T J, \mathrm{o}}^{i j, k}= & \chi_{T J, \mathrm{o}}^{i j, k}-\frac{\ell}{4 m} \chi_{T J, \mathrm{e}}^{i j, t} i q_{\perp}^{k} \\
& +\frac{\ell}{2} i q_{\perp}^{(i} \chi_{J J, \mathrm{e}}^{j), k}+\frac{\ell^{2}}{8 m} q_{\perp}^{(i} \chi_{J J, \mathrm{o}}^{j), t} q_{\perp}^{k},
\end{aligned}
$$

and we note the relations $\chi_{T J, \mathrm{e}}^{i j, t}=\kappa_{\mathrm{e}}^{i j, k} i q_{k}, \chi_{J J, \mathrm{o}}^{j, t}=$ $\sigma_{\mathrm{o}} q_{\perp}^{j}, \chi_{J J, \mathrm{e}}^{j, k}=\rho_{\mathrm{e}} q_{\perp}^{j} q_{\perp}^{k}$, between the above susceptibilities, the response functions $\kappa_{\mathrm{e}}, \sigma_{\mathrm{o}}$, and the London diamagnetic response $\rho_{\mathrm{e}}$.

\section{DISCUSSION}

Equations (19) and (22) are the main results of this paper. They rely on the SSB pattern Eq. (2) but not on Galilean symmetry. Equation (22) expresses $\tilde{\eta}_{\mathrm{o}}$ as a bulk observable of CSFs, which we refer to as the improved odd viscosity. According to Eq. (19), the leading term in the expansion of $\tilde{\eta}_{\mathrm{o}}(0, \mathbf{q})$ around $\mathbf{q}=\mathbf{0}$ is fixed by $c$. Since this leading term occurs at second order in $\mathbf{q}$, to extract $c$ one needs to measure $\sigma_{\mathrm{o}}, \chi_{\mathrm{e}}$, and $\eta_{\mathrm{o}}$, at zeroth, first, and second order, respectively. In a Galilean invariant system, Eqs. (19) and (22) imply Eqs. (20) and (23), respectively, which, in turn, show that $c$ can be extracted in an experiment where $U(1)_{N}$ fields and strain are applied, and the resulting number current and density are measured. In particular, a measurement of the stress tensor is not required. Since $U(1)_{N}$ fields can be applied in Galilean invariant fluids by tilting and rotating the sample, we believe that a bulk measurement of the boundary central charge, through Eqs. (20) and (23), is within reach of existing experimental techniques.

Finally, we comment on the implications of our results to $\mathrm{QH}$ physics. The problem of obtaining $c$ from a bulk observable has been previously studied in $\mathrm{QH}$ states, described by Eqs. (11) and (12) [37-39,46,56-63]. It was found that $c$ can only be extracted if $\operatorname{var} s=\overline{s^{2}}-\bar{s}^{2}=0$, in which case 
the response to strain, at fixed $A_{\mu}-\bar{s} \omega_{\mu}$, depends purely on $c[37,56]$. This is a useful theoretical characterization, which seems challenging experimentally in light of the need to maintain the fine tuned relation $A_{\mu}=\bar{s} \omega_{\mu}$ while the strain $u_{i j}$, and therefore $\omega_{\mu}$, vary in time and space. The improved odd viscosity (22), constructed here, applies also to vars $=0 \mathrm{QH}$ states, with $\ell$ replaced by $-2 \bar{s}$, and defines a bulk observable which is determined by $c$, and whose measurement does not require such fine tuning.

\section{ACKNOWLEDGMENTS}

The authors thank Daniel Ariad for sharing and explaining the Mathematica code used in Ref. [41]. We also benefited from discussions with Ady Stern, Andrey Gromov, Barry Bradlyn, David Mross, Micha Berkooz, Ryan Thorngren, Semyon Klevtsov, Tobias Holder, and Vatsal Dwivedi. O.G. acknowledges support from the Deutsche Forschungsgemeinschaft (DFG, CRC/Transregio 183, EI 519/7-1), the Israel Science Foundation (ISF), and the European Research Council (ERC, Project LEGOTOP). C.H. is partially supported by the Spanish Grant No. MINECO-16-FPA2015-63667P, the Ramon y Cajal fellowship RYC-2012-10370, and GRUPIN18-174 research grant from Principado de Asturias. The work of S.M. is funded by the Deutsche Forschungsgemeinschaft (DFG, German Research Foundation) under Emmy Noether Programme Grant No. MO 3013/1-1 and under Germany's Excellence Strategy Grant No. EXC-2111390814868 .

\section{APPENDIX A: GEOMETRIC QUANTITIES AND THEIR PERTURBATIVE EXPANSION}

We write $E_{A}^{i}=\delta_{A}^{i}+H_{A}^{i}$ for the inverse vielbein, and expand the relevant geometric quantities in $H$. For the inverse metric $G^{i j}=E_{A}^{i} \delta^{A B} E_{B}^{j}$ and volume element $\sqrt{G}=|E|=$ $\left|\operatorname{det}\left(E_{A}^{i}\right)\right|$ we find

$$
\begin{aligned}
G^{i j} & =\delta^{i j}+2 H^{(i j)}+H_{A}^{i} H^{A j} \\
& =\delta^{i j}+\delta G^{i j}, \\
\sqrt{G} & =1-H_{A}^{A}+\frac{1}{2} H_{A}^{A} H_{B}^{B}+\frac{1}{2} H_{A}^{B} H_{B}^{A}+O\left(H^{3}\right), \\
\log \sqrt{G} & =-H_{A}^{A}+\frac{1}{2} H_{A}^{B} H_{B}^{A}+O\left(H^{3}\right),
\end{aligned}
$$

where, in expanded expressions, all index manipulations are trivial, and in particular, there is no difference between coordinate indices $i, j$ and $S O(2)_{L}$ indices $A, B$. Note that the strain used in the main text is given by $u_{i j}=\left(G_{i j}-\delta_{i j}\right) / 2=$ $-H_{(i j)}+O\left(H^{2}\right)$. We use the notation $\varepsilon^{\mu \nu \rho}$ for the totally antisymmetric (pseudo) tensor, normalized such that $\varepsilon^{x y t}=$ $1 / \sqrt{G}$, as well as $\varepsilon^{i j}=\varepsilon^{i j t}$.

The nonrelativistic spin connection used in the main text is the $S O(2)_{L}$ connection

$$
\begin{aligned}
\omega_{t} & =\frac{1}{2} \varepsilon^{A B} E_{A i} \partial_{t} E_{B}^{i} \\
& =-\frac{1}{2} \partial_{t}\left(\varepsilon^{A B} H_{A B}\right)-\frac{1}{2} \varepsilon^{A B} H_{i A} \partial_{t} H_{B}^{i}+O\left(H^{3}\right),
\end{aligned}
$$

$$
\begin{aligned}
\omega_{j} & =\frac{1}{2}\left(\varepsilon^{A B} E_{A i} \partial_{j} E_{B}^{i}-\frac{1}{E} \varepsilon^{k l} \partial_{k} G_{l j}\right) \\
& =-\frac{1}{2} \partial_{j}\left(\varepsilon^{A B} H_{A B}\right)-\partial_{\perp}^{l} H_{(l j)}-\frac{1}{2} \varepsilon^{A B} H_{i A} \partial_{j} H_{B}^{i}+O\left(H^{3}\right),
\end{aligned}
$$

where $\partial_{\perp}^{l}=\varepsilon^{l k} \partial_{k}$, which is obtained naturally within NewtonCartan geometry [23,56]. This connection is torsion-full, but has a vanishing "reduced torsion" [44]. In the main text, a term $B / 2 m$ was implicitly added to $\omega_{t}$, but here we will add it explicitly when writing expressions for $S_{\text {eff }}$ and $S_{\text {ind }}$. Such a term appears in the presence of an additional background field $E_{0}^{i}$ which couples to momentum density $P_{i}[23,44]$, and can be identified with $G^{i j} A_{j} / m$ in a Galilean invariant system, where $P_{i}=m G_{i j} J^{j}$. The Ricci scalar is given by

$$
\begin{aligned}
R & =2 \varepsilon^{i j} \partial_{i} \omega_{j} \\
& =2 \partial_{\perp}^{i} \partial_{\perp}^{j} H_{i j}+O\left(H^{2}\right) \\
& =-2\left(\partial^{i} \partial^{j}-\partial^{2} \delta^{i j}\right) H_{i j}+O\left(H^{2}\right) .
\end{aligned}
$$

\section{APPENDIX B: ODD VISCOSITY AT NONZERO WAVE VECTOR: GENERALITIES}

\section{Definition and $T$ symmetry}

We define the viscosity tensor as the linear response of stress to strain rate

$$
T^{i j}(t, \mathbf{x})=\int d t d^{2} \mathbf{x}^{\prime} \eta^{i j, k l}\left(t, \mathbf{x}, t^{\prime}, \mathbf{x}^{\prime}\right) \partial_{t^{\prime}} H_{k l}\left(t^{\prime}, \mathbf{x}^{\prime}\right),
$$

where

$$
\eta^{[i j], k l}=0=\eta^{i j,[k l]} .
$$

In a translationally invariant system we can pass to Fourier components $T^{i j}(\omega, \mathbf{q})=i \omega \eta^{i j, k l}(\omega, \mathbf{q}) H_{k l}(\omega, \mathbf{q})$. By definition, $\eta^{i j, k l}\left(t, \mathbf{x}, t^{\prime}, \mathbf{x}^{\prime}\right)$ is real, and therefore

$$
\eta^{i j, k l}(\omega, \mathbf{q})=\eta^{i j, k l}(-\omega,-\mathbf{q})^{*} .
$$

Under time reversal $T$,

$$
\eta^{i j, k l}(\omega, \mathbf{q}) \mapsto \eta_{T}^{i j, k l}(\omega, \mathbf{q})=\eta^{k l, i j}(\omega,-\mathbf{q}) .
$$

The even and odd viscosities are then defined by $\eta_{\mathrm{e}, \mathrm{o}}=$ $\left(\eta \pm \eta_{T}\right) / 2$, and satisfy $\left(\eta_{\mathrm{e}, \mathrm{o}}\right)_{T}= \pm \eta_{\mathrm{e}, \mathrm{o}}$. More explicitly,

$$
\begin{aligned}
& \eta_{\mathrm{e}}^{i j, k l}(\omega, \mathbf{q})=+\eta_{\mathrm{e}}^{k l, i j}(\omega,-\mathbf{q}), \\
& \eta_{\mathrm{o}}^{i j, k l}(\omega, \mathbf{q})=-\eta_{\mathrm{o}}^{k l, i j}(\omega,-\mathbf{q}) .
\end{aligned}
$$

We will see below that in isotropic (or $S O(2)$ invariant) systems $\eta$ is even in $\mathbf{q}$, so that

$$
\begin{aligned}
& \eta_{\mathrm{e}}^{i j, k l}(\omega, \mathbf{q})=+\eta_{\mathrm{e}}^{k l, i j}(\omega, \mathbf{q}), \\
& \eta_{\mathrm{o}}^{i j, k l}(\omega, \mathbf{q})=-\eta_{\mathrm{o}}^{k l, i j}(\omega, \mathbf{q}),
\end{aligned}
$$

which is identical to the definition of $\eta_{\mathrm{e}, \mathrm{o}}$ at $\mathbf{q}=0$ [1-5].

\section{2. $S O(2)$ and $P$ symmetries}

Complex tensors satisfying Eqs. (B2) and (B7), in two spatial dimensions, form a vector space $V \cong \mathbb{C}^{3}$ which can 
be spanned by [2]

$$
\sigma^{a b}=2 \sigma^{[a} \otimes \sigma^{b]}, \quad a, b=0, x, z,
$$

where $\sigma^{x}, \sigma^{z}$ are the symmetric Pauli matrices, and $\sigma^{0}$ is the identity matrix. Thus, every odd viscosity tensor can be written as

$$
\eta_{\mathrm{o}}(\omega, \mathbf{q})=\eta_{x z}(\omega, \mathbf{q}) \sigma^{x z}+\eta_{x 0}(\omega, \mathbf{q}) \sigma^{x 0}+\eta_{z 0}(\omega, \mathbf{q}) \sigma^{z 0},
$$

with complex coefficients $\eta_{a b}(\omega, \mathbf{q})$. Under a rotation $R=$ $e^{i \alpha\left(i \sigma^{y}\right)} \in S O(2)$ the metric perturbation and stress tensor transform as

$$
\begin{aligned}
& H_{i j}(\omega, \mathbf{q}) \mapsto R_{i}^{k} R_{j}^{l} H_{k l}\left(\omega, R^{-1} \cdot \mathbf{q}\right), \\
& T^{i j}(\omega, \mathbf{q}) \mapsto R_{k}^{i} R_{k}^{j} T^{k l}\left(\omega, R^{-1} \cdot \mathbf{q}\right),
\end{aligned}
$$

where $(R \cdot \mathbf{q})^{i}=R_{j}^{i} q^{j}$. The same transformation rules apply for $R \in O(2)$, which defines the parity transformation $P$, in flat space. It follows that

$$
\eta^{i j, k l}(\omega, \mathbf{q}) \mapsto R_{i^{\prime}}^{i} R_{j^{\prime}}^{j} R_{k^{\prime}}^{k} R_{l^{\prime}}^{l} \eta^{i^{\prime} j^{\prime}, k^{\prime} l^{\prime}}\left(\omega, R^{-1} \cdot \mathbf{q}\right)
$$

under $O(2)$, which is compatible with Eq. (B2), and the decomposition $\eta=\eta_{\mathrm{o}}+\eta_{\mathrm{e}}$. In particular, Eq. (B11) shows that the viscosity tensor is $P$-even, or more accurately, a tensor under $P$ rather than a pseudo-tensor. In an $S O(2)$-invariant system, the viscosity tensor will also be $S O(2)$-invariant

$$
\eta^{i j, k l}(\omega, \mathbf{q})=R_{i^{\prime}}^{i} R_{j^{\prime}}^{j} R_{k^{\prime}}^{k} R_{l^{\prime}}^{l} \eta^{i^{\prime} j^{\prime}, k^{\prime} l^{\prime}}\left(\omega, R^{-1} \cdot \mathbf{q}\right), \quad R \in S O(2) .
$$

Note that this holds even when $S O(2)$ symmetry is spontaneously broken, as in $\ell$-wave SFs. At $\mathbf{q}=\mathbf{0}$, there is a unique tensor satisfying Eq. (B12), namely,

$$
\left(\sigma^{x z}\right)^{i j, k l}=-\frac{1}{2}\left(\varepsilon^{i k} \delta^{j l}+\varepsilon^{j k} \delta^{i l}+\varepsilon^{i l} \delta^{j k}+\varepsilon^{j l} \delta^{i k}\right),
$$

leaving a single odd viscosity coefficient $\eta_{x z}(\omega)=\eta_{\mathrm{o}}^{(1)}(\omega)$ [1-5].

A nonzero $\mathbf{q}$, however, along with the tensors $\delta^{i j}$ and $\varepsilon^{i j}$, can be used to construct additional $S O(2)$-invariant odd viscosity tensors, beyond $\sigma^{x z}$. From the data $\mathbf{q}, \delta^{i j}, \varepsilon^{i j}$, three linearly independent, symmetric, rank-2 tensors can be constructed, which we take to be

$$
\begin{aligned}
& \left(\tau^{0}\right)^{i j}=q^{2} \delta^{i j}, \\
& \left(\tau^{x}\right)^{i j}=-2 q_{\perp}^{(i} q^{j)} / q^{2}, \\
& \left(\tau^{z}\right)^{i j}=2 q^{i} q^{j} / q^{2}-\delta^{i j},
\end{aligned}
$$

where $q_{\perp}^{i}=\varepsilon^{i j} q_{j}$. The notation above is due to the relation

$$
\begin{aligned}
\left(\begin{array}{l}
\tau^{x} \\
\tau^{z}
\end{array}\right) & =\left(\begin{array}{cc}
\cos 2 \theta & -\sin 2 \theta \\
\sin 2 \theta & \cos 2 \theta
\end{array}\right)\left(\begin{array}{l}
\sigma^{x} \\
\sigma^{z}
\end{array}\right) \\
& =\frac{1}{q^{2}}\left(\begin{array}{cc}
q_{x}^{2}-q_{y}^{2} & -2 q_{x} q_{y} \\
2 q_{x} q_{y} & q_{x}^{2}-q_{y}^{2}
\end{array}\right)\left(\begin{array}{l}
\sigma^{x} \\
\sigma^{z}
\end{array}\right),
\end{aligned}
$$

where $\theta=\arg (\mathbf{q})$, so that $\tau^{x}, \tau^{z}$ are a rotated version of $\sigma^{x}, \sigma^{z}$. Moreover, all three $\tau$ s are $S O(2)$-invariant, $\tau^{i j}(\mathbf{q})=$ $R_{i^{\prime}}^{i} R_{j^{\prime}}^{j} i^{i^{\prime} j^{\prime}}\left(R^{-1} \cdot \mathbf{q}\right)$, and can therefore be used to construct three $S O(2)$-invariant odd viscosity tensors

$$
\tau^{a b}=2 \tau^{[a} \otimes \tau^{b]}, \quad a, b=0, x, z,
$$

which form a basis for $V$. Any odd viscosity tensor (at $\mathbf{q} \neq \mathbf{0}$ ) can then be written as

$$
\eta_{\mathrm{o}}(\omega, \mathbf{q})=\eta_{\mathrm{o}}^{(1)}(\omega, \mathbf{q}) \tau^{x z}+\eta_{\mathrm{o}}^{(2)}(\omega, \mathbf{q}) \tau^{0 x}+\eta_{\mathrm{o}}^{(3)}(\omega, \mathbf{q}) \tau^{0 z} .
$$

Furthermore, for an $S O(2)$-invariant $\eta_{\mathrm{o}}$, the coefficients $\eta_{\mathrm{o}}^{(1)}, \eta_{\mathrm{o}}^{(2)}, \eta_{\mathrm{o}}^{(3)}$ depend on $\mathbf{q}$ through its norm, owing to the $S O(2)$-invariance of $\tau^{a b}$. We therefore arrive at the general form of an $S O(2)$-invariant odd viscosity tensor,

$$
\eta_{\mathrm{o}}(\omega, \mathbf{q})=\eta_{\mathrm{o}}^{(1)}\left(\omega, q^{2}\right) \tau^{x z}+\eta_{\mathrm{o}}^{(2)}\left(\omega, q^{2}\right) \tau^{0 x}+\eta_{\mathrm{o}}^{(3)}\left(\omega, q^{2}\right) \tau^{0 z} .
$$

In particular, we see that $\eta_{\mathrm{o}}$ is even in $\mathbf{q}$ (and the same applies also to the even viscosity $\eta_{\mathrm{e}}$ ). To determine the small $\omega, \mathbf{q}$ behavior of the coefficients we change to the q-independent basis of $\sigma \mathrm{s}$,

$$
\begin{aligned}
\eta_{\mathrm{o}}(\omega, \mathbf{q})= & \eta_{\mathrm{o}}^{(1)}\left(\omega, q^{2}\right) \sigma^{x z}+\left[\eta_{\mathrm{o}}^{(2)}\left(\omega, q^{2}\right)\left(q_{x}^{2}-q_{y}^{2}\right)\right. \\
& \left.+\eta_{\mathrm{o}}^{(3)}\left(\omega, q^{2}\right)\left(2 q_{x} q_{y}\right)\right] \sigma^{0 x}+\left[\eta_{\mathrm{o}}^{(2)}\left(\omega, q^{2}\right)\left(-2 q_{x} q_{y}\right)\right. \\
& \left.+\eta_{\mathrm{o}}^{(3)}\left(\omega, q^{2}\right)\left(q_{x}^{2}-q_{y}^{2}\right)\right] \sigma^{0 z} .
\end{aligned}
$$

In gapped systems (such as $\mathrm{QH}$ states) $\eta_{\mathrm{o}}$ will be regular around $\omega=0=q$, and so will the coefficients $\eta_{\mathrm{o}}^{(1)}, \eta_{\mathrm{o}}^{(2)}, \eta_{\mathrm{o}}^{(3)}$. In gapless systems (such as $\ell$-wave $\mathrm{SFs}$ ) there will be a singularity at $\omega=0=q$, but the limit $q \rightarrow 0$ at $\omega \neq 0$ will be regular. In both cases, the limit $q \rightarrow 0$ at $\omega \neq 0$ of Eq. (B19) reduces to the known result $\eta_{\mathrm{o}}(\omega, \mathbf{0})=\eta_{\mathrm{o}}^{(1)}(\omega, 0) \sigma^{x z}[1-5]$.

\section{PT symmetry}

The combination $P T$ of parity and time reversal is a symmetry in any system in which $T$ is broken (perhaps spontaneously) due to some kind of angular momentum, as in $\mathrm{QH}$ states, $\ell$-wave SFs, and active chiral fluids [6]. Here we consider the implications of $P T$ symmetry on Eq. (B19).

From the definition Eq. (B14) it is clear that $\tau^{0}$ and $\tau^{z}$ are $P$-even, while $\tau^{x}$ is $P$-odd. Therefore, $\tau^{x z}, \tau^{0 x}$ are $P$-odd while $\tau^{0 z}$ is $P$-even (and all three are $T$-even). Since $\eta_{\mathrm{o}}$ is $T$-odd and $P$-even, and using Eq. (B18), it follows that $\eta_{\mathrm{o}}^{(1)}$ and $\eta_{\mathrm{o}}^{(2)}$ are $P, T$-odd, while $\eta_{\mathrm{o}}^{(3)}$ is $T$-odd but $P$-even. In particular, $\eta_{\mathrm{o}}^{(3)}$ is $P T$-odd, and must vanish in $P T$-symmetric systems. The odd viscosity tensor in $S O(2)$ and $P T$ symmetric systems is therefore given by

$$
\begin{aligned}
\eta_{\mathrm{o}}(\omega, \mathbf{q})= & \eta_{\mathrm{o}}^{(1)}\left(\omega, q^{2}\right) \sigma^{x z} \\
& +\eta_{\mathrm{o}}^{(2)}\left(\omega, q^{2}\right)\left[\left(q_{x}^{2}-q_{y}^{2}\right) \sigma^{0 x}-2 q_{x} q_{y} \sigma^{0 z}\right] .
\end{aligned}
$$

This form is confirmed by previous results for $\mathrm{QH}$ states [38], and by the results presented in Sec.V for CSFs. The same form is obtained at $\mathbf{q}=\mathbf{0}$, but in the presence of vector, or pseudovector, anisotropy $\mathbf{b}$, in which case we find

$$
\begin{aligned}
\eta_{\mathrm{o}}(\omega)= & \eta_{\mathrm{o}}^{(1)}(\omega) \sigma^{x z} \\
& +\eta_{\mathrm{o}}^{(2)}(\omega)\left[\left(b_{x}^{2}-b_{y}^{2}\right) \sigma^{0 x}-2 b_{x} b_{y} \sigma^{0 z}\right],
\end{aligned}
$$

which explains the tensor structure found in Refs. [71,72].

\section{Frequency dependence and reality conditions}

In closed and clean systems, like the $\ell$-wave SFs discussed in this paper, the viscosity can be obtained from an induced 
action

$$
\begin{aligned}
S_{\text {ind }} \supset & \frac{1}{2} \int d t d t^{\prime} d^{2} \mathbf{x} d^{2} \mathbf{x}^{\prime} H_{i j}(t, \mathbf{x}) \eta^{i j, k l} \\
& \times\left(t-t^{\prime}, \mathbf{x}-\mathbf{x}^{\prime}\right) \partial_{t^{\prime}} H_{k l}\left(t^{\prime}, \mathbf{x}^{\prime}\right) \\
= & \frac{1}{2} \int \frac{d \omega}{2 \pi} \frac{d^{2} \mathbf{q}}{(2 \pi)^{2}} H_{i j} \\
& \times(-\omega,-\mathbf{q}) i \omega \eta^{i j, k l}(\omega, \mathbf{q}) H_{k l}(\omega, \mathbf{q}) .
\end{aligned}
$$

As a result, $\eta$ satisfies the additional property,

$$
\eta^{i j, k l}(\omega, \mathbf{q})=-\eta^{k l, i j}(-\omega,-\mathbf{q})
$$

which, along with Eqs. (B7) and (B6) and the fact that $\eta$ is even in $\mathbf{q}$, implies that $\eta_{\mathrm{o}}\left(\eta_{\mathrm{e}}\right)$ is even (odd) in $\omega$,

$$
\begin{aligned}
& \eta_{\mathrm{e}}^{i j, k l}(\omega, \mathbf{q})=-\eta_{\mathrm{e}}^{i j, k l}(-\omega, \mathbf{q}), \\
& \eta_{\mathrm{o}}^{i j, k l}(\omega, \mathbf{q})=+\eta_{\mathrm{o}}^{i j, k l}(-\omega, \mathbf{q}) .
\end{aligned}
$$

This result, along with Eq. (B3) and the fact that $\eta$ is even in $\mathbf{q}$, implies that $\eta_{\mathrm{o}}\left(\eta_{\mathrm{e}}\right)$ is real (imaginary),

$$
\begin{aligned}
& \eta_{\mathrm{e}}^{i j, k l}(\omega, \mathbf{q}) \in i \mathbb{R}, \\
& \eta_{\mathrm{o}}^{i j, k l}(\omega, \mathbf{q}) \in \mathbb{R} .
\end{aligned}
$$

These general properties are satisfied by the odd viscosity tensor computed in this paper. These are also compatible with the examples worked out in Ref. [3], as well with viscosityconductivity relations that hold in Galilean invariant systems (in conjugation with known properties of the conductivity) $[3,21,45]$.

We note that some care is required when interpreting Eqs. (B24) and (B25) around singularities of $\eta$. For example, the first equation in Eq. (B24) naively implies that $\eta_{\mathrm{e}}(0, \mathbf{q})=$ 0 , which in particular implies that the bulk and shear viscosities $\eta_{\mathrm{e}}(0, \mathbf{0})=\zeta \sigma^{0} \otimes \sigma^{0}+\eta^{\mathrm{s}}\left(\sigma^{x} \otimes \sigma^{z}+\sigma^{z} \otimes \sigma^{x}\right)$ vanish in the closed, clean case. This, however, is not quite correct, due to a possible singularity of $\eta_{\mathrm{e}}$ at $\omega=0$, as well as the usual infinitesimal imaginary part of $\omega$ required to obtain the retarded response. For example, for free fermions, Ref. [3] finds $\eta^{\mathrm{s}}(\omega, \mathbf{0}) \sim \frac{i}{\omega+i \epsilon}=\pi \delta(\omega)+i \mathrm{PV} \frac{1}{\omega}$ (where $\mathrm{PV}$ is the principle value), which has an infinite real part at $\omega=0$, in analogy with the Drude behavior of the conductivity.

\section{Odd viscosity from Gaussian integration: A technical result}

We now restrict attention to CSFs. The effective Lagrangian, perturbatively expanded to second order, and in the absence of the $U(1)$ background, takes the form

$$
\mathcal{L}_{\text {eff }}=\frac{1}{2} \theta \mathcal{G}^{-1} \theta+\mathcal{V} \theta+\mathcal{C},
$$

where the Green's function $\mathcal{G}$ is independent of $H$, the vertex $\mathcal{V}$ is linear in $H$, and the contact term $\mathcal{C}$ is quadratic in $H$. Performing Gaussian integration over $\theta$ yields the induced Lagrangian

$$
\mathcal{L}_{\text {ind }}=-\frac{1}{2} \mathcal{V G} \mathcal{V}+\mathcal{C}
$$

and comparing with Eq. (B22) one can read off $\eta_{\mathrm{o}}$. In Appendix $\mathrm{C}$ we write explicit expressions for a Galilean invariant $\mathcal{L}_{\text {eff }}$, which we then expand to obtain explicit expressions for $\mathcal{G}^{-1}, \mathcal{V}, \mathcal{C}$. Appendix $\mathrm{D}$ then describes the resulting $\mathcal{L}_{\text {ind }}$. Here we take a complementary approach and obtain the general form of $\eta_{\mathrm{o}}$ from Eq. (B27), using the formalism developed above, based only on $S O(2)$ and $P T$ symmetries.

The motivation for the analysis in this Appendix is the following. The power counting Eq. (8) is designed such that the $O\left(p^{n}\right)$ Lagrangian $\mathcal{L}_{n} \subset \mathcal{L}_{\text {eff }}$ produces $O\left(p^{n}\right)$ contributions to $\mathcal{L}_{\text {ind }}$. Therefore, naively, one expects the $O\left(q^{2}\right)$ odd viscosity to depend on $\mathcal{L}_{0}, \mathcal{L}_{2}$, and $\mathcal{L}_{3}$ (since $\mathcal{L}_{1}=0$ ). Using the notation $\eta_{\mathrm{o}}=\eta_{\mathcal{V}}+\eta_{\mathcal{C}}$ for the parts of $\eta_{\mathrm{o}}$ due to $-\mathcal{V G \mathcal { V }} / 2$ and $\mathcal{C}$, respectively, the result of this Appendix is that $\eta_{\mathcal{V}}$, to $O\left(q^{2}\right)$, is actually independent of $\mathcal{L}_{3}$.

We now describe the details. For $\eta_{\mathcal{C}}$, we cannot do better than the general discussion thus far-it is given by Eq. (B19), with $\eta_{\mathrm{o}}^{(3)}=0$, and both $\eta_{\mathrm{o}}^{(1)}, \eta_{\mathrm{o}}^{(2)}$ are real and regular at $\omega=0=q$, since $\mathcal{L}_{\text {eff }}$, and $\mathcal{C}$ in particular, are obtained by integrating out gapped degrees of freedom (the Higgs modes and the fermion $\psi$ ). For $\eta_{\mathcal{V}}$, however, we can do better. We first write more explicitly

$$
\begin{aligned}
\theta \mathcal{G}^{-1} \theta & =\frac{1}{2} \theta(-\omega,-\mathbf{q}) \mathcal{G}^{-1}(\omega, \mathbf{q}) \theta(\omega, \mathbf{q}), \\
\mathcal{V} \theta & =\theta(-\omega,-\mathbf{q}) V^{i j}(\omega, \mathbf{q}) H_{i j}(\omega, \mathbf{q}) .
\end{aligned}
$$

Based on $S O(2)$ and $P T$ symmetries, the objects $\mathcal{G}^{-1}, V^{i j}$ take the forms

$$
\begin{aligned}
\mathcal{G}^{-1}(\omega, \mathbf{q})= & D\left(\omega^{2}, q^{2}\right) \\
V^{i j}(\omega, \mathbf{q})= & i \omega a\left(\omega^{2}, q^{2}\right)\left(\rho^{0}\right)^{i j}+i \omega b\left(\omega^{2}, q^{2}\right)\left(\rho^{z}\right)^{i j} \\
& +s_{\theta} c\left(\omega^{2}, q^{2}\right)\left(\rho^{x}\right)^{i j}
\end{aligned}
$$

where

$$
\begin{aligned}
& \left(\rho^{0}\right)^{i j}=\delta^{i j}, \\
& \left(\rho^{x}\right)^{i j}=q_{\perp}^{(i} q^{j)}, \\
& \left(\rho^{z}\right)^{i j}=q^{i} q^{j},
\end{aligned}
$$

are, in this context, more convenient than the $\tau$ s Eq. (B14), and $a, b, c, D$ are general functions of their arguments which are $P, T$-even, real, and regular at $\omega=0=q$, as follows from the same properties of $\mathcal{L}_{\text {eff }}$. In particular, we will use the following expansions

$$
\begin{aligned}
& a\left(0, q^{2}\right)=a_{0}+a_{1} q^{2}+O\left(q^{4}\right), \\
& b\left(0, q^{2}\right)=b_{0}+O\left(q^{2}\right), \\
& c\left(0, q^{2}\right)=c_{0}+c_{1} q^{2}+O\left(q^{4}\right), \\
& D\left(0, q^{2}\right)=D_{1} q^{2}+D_{2} q^{4}+O\left(q^{6}\right),
\end{aligned}
$$

where $D_{0}=0$ because $\theta$ enters $\mathcal{L}_{\text {eff }}$ only through its derivatives. The odd viscosity $\eta \mathcal{V}$ is then given by

$$
\eta_{\mathcal{V}}(\omega, \mathbf{q})=-\frac{1}{2 i \omega} \frac{V(-\omega,-\mathbf{q}) \otimes V(\omega, \mathbf{q})-V(\omega, \mathbf{q}) \otimes V(-\omega,-\mathbf{q})}{D(\omega, \mathbf{q})}=\frac{2 s_{\theta} c\left(\omega^{2}, q^{2}\right)}{D\left(\omega^{2}, q^{2}\right)}\left[a\left(\omega^{2}, q^{2}\right) \rho^{0 x}+b\left(\omega^{2}, q^{2}\right) \rho^{z x}\right]
$$


which is of the form Eq. (B19), with $\eta_{\mathrm{o}}^{(3)}=0$ and

$$
\begin{aligned}
& \eta_{\mathcal{V}}^{(1)}\left(\omega, q^{2}\right)=-\frac{s_{\theta} c\left(\omega^{2}, q^{2}\right)}{2 D\left(\omega^{2}, q^{2}\right)} b\left(\omega^{2}, q^{2}\right) q^{4} \\
& \eta_{\mathcal{V}}^{(2)}\left(\omega, q^{2}\right)=\frac{s_{\theta} c\left(\omega^{2}, q^{2}\right)}{D\left(\omega^{2}, q^{2}\right)}\left[a\left(\omega^{2}, q^{2}\right)+b\left(\omega^{2}, q^{2}\right) q^{2}\right] .
\end{aligned}
$$

Setting $\omega=0$ and expanding in $q$, we find

$$
\begin{aligned}
\eta_{\mathcal{V}}^{(1)}\left(0, q^{2}\right)= & -\frac{s_{\theta} c_{0} b_{0}}{2 D_{1}} q^{2}+O\left(q^{4}\right), \\
\eta_{\mathcal{V}}^{(2)}\left(0, q^{2}\right)= & \frac{s_{\theta}}{D_{1}}\left[a_{0} c_{0} q^{-2}+\left(a_{0} c_{1}+a_{1} c_{0}\right.\right. \\
& \left.\left.+b_{0} c_{0}-a_{0} c_{0} \frac{D_{2}}{D_{1}}\right)\right]+O\left(q^{2}\right) .
\end{aligned}
$$

Having identified the coefficients $a_{0}, a_{1}, b_{0}, c_{0}, c_{1}, D_{1}, D_{2}$ that determine $\eta_{\mathcal{V}}$ to $O\left(q^{2}\right)$, we now determine the order in the derivative expansion of $\mathcal{L}_{\text {eff }}$ in which these enter. Explicitly, the above coefficients are defined by

$$
\begin{aligned}
\mathcal{L}_{\text {eff }} \supset & \frac{1}{2} \theta(-\omega,-\mathbf{q})\left(D_{1} q^{2}+D_{2} q^{4}\right) \theta(\omega, \mathbf{q}) \\
& +\theta(-\omega,-\mathbf{q})\left[i \omega\left(a_{0}+a_{1} q^{2}\right) \delta^{i j}+i \omega b_{0} q^{i} q^{j}\right. \\
& \left.+s_{\theta}\left(c_{0}+c_{1} q^{2}\right) q^{i} q_{\perp}^{j}\right] H_{i j}(\omega, \mathbf{q}) .
\end{aligned}
$$

We see that $c_{1}$ enters $\mathcal{L}_{\text {eff }}$ at $O\left(p^{3}\right)$, while all other coefficients enter at a lower order, and come from $\mathcal{L}_{0}, \mathcal{L}_{2}$. In particular, $\eta_{\mathcal{V}}^{(1)}$ in Eq. (B34) is independent of $\mathcal{L}_{3}$. Even though $c_{1}$ is the coefficient of an $O\left(p^{3}\right)$ term, it is actually due to $\mathcal{L}_{2}$. Using Eq. (A2) we identify $c_{0} \theta q^{2} q^{i} q_{\perp}^{j} H_{i j}=-s_{\theta} c_{1} \partial^{i} \theta \partial^{2} \omega_{i}$, which must be a part of

$$
\frac{c_{1}}{2}\left(\partial_{i} \theta-A_{i}-s_{\theta} \omega_{i}\right) \partial^{2}\left(\partial_{i} \theta-A_{i}-s_{\theta} \omega_{i}\right) .
$$

This is an $O\left(p^{2}\right)$ term, and in fact comes from $\mathcal{L}_{2}^{(2)} \subset \mathcal{L}_{2}$, see Eq. (C7). Thus, both $\eta_{\mathcal{V}}^{(1)}, \eta_{\mathcal{V}}^{(2)}$ in Eq. (B34) are completely independent of $\mathcal{L}_{3}$.

\section{APPENDIX C: EFFECTIVE ACTION AND ITS PERTURBATIVE EXPANSION}

\section{Zeroth order}

It is useful to write the zeroth order scalar $X$ as

$$
X=\left(\partial_{t} \theta-\mathcal{A}_{t}-\frac{s_{\theta}}{2 m} B\right)-\frac{1}{2 m} G^{i j}\left(\partial_{i} \theta-\mathcal{A}_{i}\right)\left(\partial_{j} \theta-\mathcal{A}_{j}\right),
$$

where

$$
\mathcal{A}_{\mu}=A_{\mu}+s_{\theta} \omega_{\mu} .
$$

We will also use $\mathcal{B}=B+\frac{s_{\theta}}{2} R, \mathcal{E}_{i}=E_{i}+s_{\theta} E_{\omega, i}$ for the magnetic and electric fields obtained from $\mathcal{A}_{\mu}$, where $E_{\omega, i}=$ $\partial_{t} \omega_{i}-\partial_{i} \omega_{t}$. Expanding $\mathcal{L}_{0}=P(X)$ to second order in the fields, one finds (up to total derivatives)

$$
\begin{aligned}
\sqrt{G} \mathcal{L}_{0}= & \frac{1}{2} \frac{n_{0}}{m} \theta\left[\partial^{2}-c_{s}^{-2} \partial_{t}^{2}\right] \theta+\left[-\frac{n_{0}}{m}\left(\partial_{i} \mathcal{A}^{i}-c_{s}^{-2} \partial_{t}\left(\mathcal{A}_{t}+\frac{s_{\theta}}{2 m} B\right)\right)-n_{0} \partial_{t} \sqrt{G}\right] \theta \\
& +\left[-n_{0} \sqrt{G} \mathcal{A}_{t}-\frac{1}{2} \frac{n_{0}}{m}\left(\mathcal{A}^{2}-c_{s}^{-2}\left(\mathcal{A}_{t}+\frac{s_{\theta}}{2 m} B\right)^{2}\right)+P_{0} \sqrt{G}\right]=\frac{1}{2} \theta \mathcal{G}^{-1} \theta+\mathcal{V} \theta+\mathcal{C},
\end{aligned}
$$

where $\partial^{2}=\partial^{i} \partial_{i}, \mathcal{A}^{2}=\mathcal{A}_{i} \mathcal{A}^{i}$, and we defined the inverse Green's function $\mathcal{G}^{-1}$, vertex $\mathcal{V}$, and contact terms $\mathcal{C}$, respectively. These are used in Appendix D below to obtain $S_{\text {ind }}$.

In Eq. (C3), the geometric objects $\sqrt{G}$ and $\omega_{\mu}$ should be interpreted as expanded to the required order according to Eqs. (A1) and (A2). In particular, the term $-n_{0} \sqrt{G} \mathcal{A}_{t}$ includes $-s_{\theta} n_{0} \sqrt{G} \omega_{t}$, which produces the leading contribution to $\eta_{\mathrm{o}}^{(1)}$. To see this, we expand

$$
\sqrt{G} \omega_{t}=-\frac{1}{2} \partial_{t}\left(\varepsilon^{A B} H_{A B}\right)+\frac{1}{2} \partial_{t}\left(\varepsilon^{A B} H_{A B}\right) H_{i}^{i}-\frac{1}{2} \varepsilon^{A B} H_{i A} \partial_{t} H_{B}^{i}+O\left(H^{3}\right)=-\frac{1}{2} \partial_{t}\left(\varepsilon^{A B} H_{A B}\right)-\frac{1}{2} \varepsilon^{A B} H_{A i} \partial_{t} H_{B}^{i}+O\left(H^{3}\right),
$$

which is identical to the expansion Eq. (A1) of $\omega_{t}$, apart from $H_{i A} \leftrightarrow H_{A i}$. Ignoring total derivatives, this reduces to

$$
\sqrt{G} \mathcal{L}_{0} \supset-s_{\theta} n_{0} \sqrt{G} \omega_{t}=-\frac{1}{2} s_{\theta} n_{0}\left[\partial_{t}\left(\varepsilon^{A B} H_{A B}\right) H_{i}^{i}-\varepsilon^{A B} \delta^{i j} H_{(A i)} \partial_{t} H_{(B j)}\right]+O\left(H^{3}\right)=\frac{1}{2} s_{\theta} n_{0} \varepsilon^{A B} H_{A i} \partial_{t} H_{B}^{i}+O\left(H^{3}\right) .
$$

Comparing with Eqs. (B13) and (B22), the second term in the second line corresponds to $\eta_{\mathrm{o}}^{(1)}=-s_{\theta} n_{0} / 2$. The first term in the second line depends on the anti-symmetric part of $H$, and shows that the full expression, Eq. (C5), actually corresponds to a torsional Hall (or odd) viscosity $[69,74] \zeta_{H}=-s_{\theta} n_{0}$, which can be read off from the third line. The appearance of the torsional Hall viscosity at the level of $S_{\text {eff }}$ (but not at the level of $S_{\text {ind }}$, see Appendix D) can be understood from the mapping of [25] of the $p$-wave SF to a Majorana spinor in Riemann-Cartan space-time.

\section{Second order}

The full expression for $\mathcal{L}_{2}$ is given by $\mathcal{L}_{2}=\sum_{i=1}^{6} \mathcal{L}_{2}^{(i)}$, where [42]

$$
\begin{aligned}
\mathcal{L}_{2}^{(1)}= & F_{1}(X) R, \quad \mathcal{L}_{2}^{(2)}=F_{2}(X)\left(m K_{i}^{i}-\nabla^{2} \theta\right)^{2}, \\
\mathcal{L}_{2}^{(3)}= & F_{3}(X)\left\{-m^{2}\left(G^{i j} \partial_{t} K_{i j}-K^{i j} K_{i j}\right)-m \nabla_{i} E^{i}+\frac{1}{4} F^{i j} F_{i j}\right. \\
& \left.+2 m\left[\partial_{i} K_{j}^{j}-\nabla^{j}\left(K_{j i}+\frac{1}{2 m} F_{j i}\right)\right] \nabla^{i} \theta+R_{i j} \nabla_{i} \theta \nabla_{j} \theta\right\},
\end{aligned}
$$




$$
\begin{aligned}
& \mathcal{L}_{2}^{(4)}=F_{4}(X) G^{i j} \partial_{i} X \partial_{j} X, \quad \mathcal{L}_{2}^{(5)}=F_{5}(X)\left[\left(\partial_{t}-\frac{1}{m} \nabla^{i} \theta \partial_{i}\right) X\right]^{2}, \\
& \mathcal{L}_{2}^{(6)}=F_{6}(X)\left(m K_{i}^{i}-\nabla^{2} \theta\right)\left[\left(\partial_{t}-\frac{1}{m} \nabla^{i} \theta \partial_{i}\right) X\right] .
\end{aligned}
$$

The terms $\mathcal{L}_{2}^{(5)}$ and $\mathcal{L}_{2}^{(6)}$ were not written explicitly in [42] because, on shell (on the equation of motion for $\theta$ ), they are proportional to $\mathcal{L}_{2}^{(4)}$ up to $O\left(p^{4}\right)$ corrections, and can therefore be eliminated by a redefinition of $F_{4}$. However, for the purpose of comparing the general $S_{\text {eff }}$ with the microscopic expression, Eq. (E20), it is convenient to work off shell and keep all terms explicit.

Specializing to $2+1$ dimensions and expanding to second order in fields, one finds

$$
\begin{aligned}
\sqrt{G} \mathcal{L}_{2}^{(1)}= & F_{1}^{\prime}(\mu) R\left(\partial_{t} \theta-\mathcal{A}_{t}-\frac{s_{\theta}}{2 m} B\right), \\
\sqrt{G} \mathcal{L}_{2}^{(2)}= & F_{2}(\mu)\left[-m^{2} H_{i}^{i} \partial_{t}^{2} H_{j}^{j}+2 m \partial_{t} H_{k}^{k} \partial^{j}\left(\partial_{j} \theta-\mathcal{A}_{j}\right)\right. \\
& \left.-\left(\partial_{i} \theta-\mathcal{A}_{i}\right) \partial^{i} \partial^{j}\left(\partial_{i} \theta-\mathcal{A}_{i}\right)\right], \\
\sqrt{G} \mathcal{L}_{2}^{(3)}= & F_{3}(\mu)\left(m^{2} H^{(i j)} \partial_{t}^{2} H_{(i j)}+\frac{1}{2} B^{2}\right. \\
& \left.-2 m \varepsilon^{i j} \omega_{i} \partial_{t}\left(\partial_{j} \theta-\mathcal{A}_{j}\right)-B \mathcal{B}\right) \\
& +F_{3}^{\prime}(\mu)\left(\partial_{t} \theta-\mathcal{A}_{t}-\frac{s_{\theta}}{2 m} B\right)\left(m^{2} \partial_{t}^{2} H_{i}^{i}-m \partial_{i} E^{i}\right), \\
\sqrt{G} \mathcal{L}_{2}^{(4)}= & -F_{4}(\mu)\left(\partial_{t} \theta-\mathcal{A}_{t}-\frac{s_{\theta}}{2 m} B\right) \partial^{2}\left(\partial_{t} \theta-\mathcal{A}_{t}-\frac{s_{\theta}}{2 m} B\right), \\
\sqrt{G} \mathcal{L}_{2}^{(5)}= & -F_{5}(\mu)\left(\partial_{t} \theta-\mathcal{A}_{t}-\frac{s_{\theta}}{2 m} B\right) \partial_{t}^{2}\left(\partial_{t} \theta-\mathcal{A}_{t}-\frac{s_{\theta}}{2 m} B\right), \\
\sqrt{G} \mathcal{L}_{2}^{(6)}= & -F_{6}(\mu)\left[m \partial_{t} H_{i}^{i}+\partial^{j}\left(\partial_{j} \theta-\mathcal{A}_{j}\right)\right] \partial_{t} \\
& \times\left(\partial_{t} \theta-\mathcal{A}_{t}-\frac{s_{\theta}}{2 m} B\right),
\end{aligned}
$$

from which one can easily extract the second order corrections to $\mathcal{G}^{-1}, \mathcal{V}, \mathcal{C}$, of Eq. (C3). Note that $\mathcal{L}_{2}^{(3)}$ includes a term $\propto \varepsilon^{i j} \omega_{i} \partial_{t} \mathcal{A}_{j}=\varepsilon^{i j} \omega_{i} \partial_{t}\left(A_{j}+s_{\theta} \omega_{j}\right)$. Comparing with Eq. (C8) below, it is clear that distinguishing $\mathcal{L}_{2}^{(3)}$ from $\mathcal{L}_{\mathrm{gCS}}$ is nontrivial. This is, in fact, the same problem of extracting the central charge from the Hall viscosity addressed in the main text, but at the level of $S_{\text {eff }}$ (where $\theta$ is viewed as a background field) rather than $S_{\text {ind }}$ (where $\theta$ has been integrated out). Accordingly, the central charge can be computed by applying Eq. (22) to the response functions obtained from $S_{\text {eff }}$. Additionally, relying on LGS, one can extract $F_{3}$ as the coefficient of $H^{(i j)} \partial_{t}^{2} H_{(i j)}$. Both approaches produce the same central charge Eq. (14) in the perturbative computation of Appendix E 5.

\section{Gravitational Chern-Simons term}

The gCS Lagrangian is given explicitly by

$$
\begin{aligned}
\mathcal{L}_{\mathrm{gCS}} & =-\frac{c}{48 \pi}\left[\left(\omega_{t}+\frac{B}{2 m}\right) R-\varepsilon^{i j} \omega_{i} \partial_{t} \omega_{j}\right] \\
& =-\frac{c}{48 \pi}\left[\omega d \omega+\frac{1}{2 m} B R\right] .
\end{aligned}
$$

Its expansion to second order in fields, using Eqs. (A1) and (A2), is

$$
\begin{aligned}
\sqrt{G} \mathcal{L}_{\mathrm{gCS}}= & -\frac{c}{48 \pi}\left[\varepsilon^{A B} H_{(A i)} \partial_{\perp}^{i} \partial_{\perp}^{j} \partial_{t} H_{(B j)}\right. \\
& \left.-\frac{1}{m} A_{i} \partial_{\perp}^{i} \partial_{\perp}^{j} \partial_{\perp}^{k} H_{(j k)}\right]
\end{aligned}
$$

As opposed to $\sqrt{G} \omega_{t}$ in Eq. (C4), the gCS term is (locally) $S O(2)_{L}$ gauge invariant, and accordingly depends only on the metric, or, within the perturbative expansion, on the symmetric part $H_{(i j)}$. From this expansion one can read off the gCS contributions to the odd viscosity $\eta_{\mathrm{o}}$ Eq. (19), and to the odd, mixed, static susceptibility $\chi_{T J, \mathrm{o}}$ Eq. (20).

\section{Additional terms at third order}

To obtain reliable results at $O\left(p^{3}\right)$ we, in principle, need the full Lagrangian $\mathcal{L}_{3}$, which includes, but is not equal to, $\mathcal{L}_{\mathrm{gCS}}$. Nevertheless, we argue that $\mathcal{L}_{3}-\mathcal{L}_{\mathrm{gCS}}$ does not contribute to the quantity of interest in this paper- $-\eta_{\mathrm{o}}$ to $O\left(q^{2}\right)$. We already demonstrated in Appendix B 5 that the vertex part of the odd viscosity $\eta_{\mathcal{V}}$ is independent of $\mathcal{L}_{3}$, and it remains to show that the contact term part $\eta_{\mathcal{C}}$ is independent of $\mathcal{L}_{3}-\mathcal{L}_{\mathrm{gCS}}$. We do not have a general proof, but we address this issue in two ways:

(1) Within the microscopic model Eq. (13), the perturbative computation of Appendix E 5 provides an explicit expression for $\eta_{\mathcal{C}}$, which is completely saturated by the effective action presented thus far. Thus, $\eta_{\mathcal{C}}$ is independent of $\mathcal{L}_{3}-$ $\mathcal{L}_{\mathrm{gCS}}$ in the particular realization Eq. (13).

(2) The term $\mathcal{L}_{3}$ is $P, T$-odd, and therefore vanishes in an $s$-wave SF. On the other hand, it suffices to consider the gs-wave SF where $s_{\theta}=0$ (but $\ell \neq 0$ ), since for $s_{\theta} \neq 0$ the spin connection included in $\nabla_{\mu} \theta$ will only produce $O\left(p^{4}\right)$ corrections. By contracting Galilean vectors, we were able to construct four $P, T$-odd terms in $\mathcal{L}_{3}-\mathcal{L}_{\mathrm{gCS}}$ for the gs-wave $\mathrm{SF}$,

$$
\begin{aligned}
\mathcal{L}_{3}-\mathcal{L}_{\mathrm{gCS}} \supset & \ell\left[C_{1}(X) \tilde{E}_{i} E_{\omega}^{i}+C_{2}(X) \varepsilon^{i j} \tilde{E}_{i} E_{\omega, j}\right. \\
& \left.+C_{3}(X) \partial_{i} X E_{\omega}^{j}+C_{4}(X) \varepsilon^{i j} \partial_{i} X E_{\omega, j}\right],
\end{aligned}
$$


where $\tilde{E}_{i}$ is the electric field of the improved $U(1)$ connection $\tilde{A}_{t}=A_{t}+\frac{1}{2 m} \nabla^{i} \theta \nabla_{i} \theta, \tilde{A}_{i}=\partial_{i} \theta-s_{\theta} \omega_{i}$ [21]. Perturbatively expanding these, we do not find any $O\left(q^{2}\right)$ contributions to $\eta_{\mathcal{C}}$ (or to $\eta_{\mathcal{V}}$, in accordance with Appendix B 5).

\section{APPENDIX D: INDUCED ACTION}

The arguments presented in the main text suffice to establish the quantization of $\tilde{\eta}_{\mathrm{o}}$ and $\tilde{\chi}_{T J, \mathrm{o}}$ directly from $S_{\mathrm{eff}}-$ an explicit expression for $S_{\text {ind }}$ is not required. Nevertheless, it is instructive to compute certain contributions in $S_{\text {ind }}$ to demonstrate these results explicitly and also to reproduce simpler properties of $\ell$-wave $S F$ s. Here we will compute the contribution of $\mathcal{L}_{0}+\mathcal{L}_{2}^{(1)} \subset \mathcal{L}_{\text {eff }}$ to the induced Lagrangian $\mathcal{L}_{\text {ind }}$, and, along the way, demonstrate explicitly the relation between vars $=0 \mathrm{QH}$ states and CSFs alluded to in the discussion Sec. VI.

The starting point is the induced action due to $\mathcal{L}_{0}=P(X)$, obtained from Eq. (C3). It is given by

$$
\begin{aligned}
& \mathcal{L}_{\text {ind }}=-\frac{1}{2} \mathcal{V} \mathcal{G} \mathcal{V}+\mathcal{C} \\
& =P_{0} \sqrt{G}-n_{0} \mathcal{A}_{t} \\
& +\frac{1}{2} \frac{n_{0}}{m} \frac{\mathcal{B}^{2}-c_{s}^{-2} \mathcal{E}^{2}+\frac{s_{\theta} c_{s}^{-2}}{m} \mathcal{E}^{i} \partial_{i} B-\frac{s_{\theta}^{2} c_{s}^{-2}}{4 m^{2}}(\partial B)^{2}}{\partial^{2}-c_{s}^{-2} \partial_{t}^{2}} \\
& -n_{0} \frac{m\left(\partial_{t} \sqrt{G}\right)^{2} / 2+\left(\mathcal{E}^{i}-\frac{s_{\theta}}{2 m} \partial_{i} B\right) \partial_{i} \sqrt{G}}{\partial^{2}-c_{s}^{-2} \partial_{t}^{2}} .
\end{aligned}
$$

This expression contains, rather compactly, the entire linear response of the $\ell$-wave $\mathrm{SF}$ to $O(p)$ in the derivative expansion, as well as certain $O\left(p^{2}\right)$ contributions [21], and should be interpreted as expanded to second order using Eqs. (A1) and (A2). In using Eq. (A2), one can set $H_{[A B]}=$ 0 , since $S_{\text {ind }}$ is $S O(2)_{L}$ invariant and the anti-symmetric part $H_{[A B]}$ corresponds to the $S O(2)_{L}$ phase of the vielbein $E_{A}^{i}$. Technically, $H_{[A B]}$ always appears in the combination $\partial_{\mu}\left(\theta+s_{\theta} \varepsilon^{A B} H_{A B} / 2\right) \subset \nabla_{\mu} \theta$, so that integrating out $\theta$ eliminates $H_{[A B]}$.

Note that, diagrammatically, Eq. (D1) corresponds to linear response at tree-level. Higher orders in $\theta$ will generate diagrams with $\theta$ running in loops, which can be shown to produce $O\left(p^{3}\right)$ corrections above the leading order to any observable [42], and are therefore irrelevant for the purpose of $q^{2}$ corrections to $\eta_{\mathrm{o}}$.

The $O\left(p^{0}\right)$ part of Eq. (D1) is obtained by setting $s_{\theta}=0$, as in an $s$-wave SF,

$$
\begin{aligned}
\mathcal{L}_{\text {ind }, 0}= & P_{0} \sqrt{G}-n_{0} A_{t}+\frac{1}{2} \frac{n_{0}}{m} \frac{B^{2}-c_{s}^{-2} E^{2}}{\partial^{2}-c_{s}^{-2} \partial_{t}^{2}} \\
& -n_{0} \frac{m\left(\partial_{t} \sqrt{G}\right)^{2} / 2+E^{i} \partial_{i} \sqrt{G}}{\partial^{2}-c_{s}^{-2} \partial_{t}^{2}} .
\end{aligned}
$$

The first line contains the ground-state pressure and density $P_{0}, n_{0}$, as well as the London diamagnetic function $\rho_{\mathrm{e}}=\frac{n_{0}}{m} \frac{1}{q^{2}-c_{s}^{-2} \omega^{2}}$ and the ideal Drude longitudinal conductivity $\sigma_{\mathrm{e}}=-\frac{n_{0}}{m} \frac{i \omega c_{s}^{-2}}{q^{2}-c_{s}^{-2} \omega^{2}}$ of the SF [21]. The second line contains the mixed response and mixed static susceptibility

$$
\begin{aligned}
\kappa_{\mathrm{e}}^{i j, k} & =-n_{0} \delta^{i j} \frac{i q^{k}}{q^{2}-c_{s}^{-2} \omega^{2}}, \\
\chi_{T J, \mathrm{e}}^{i j, t} & =n_{0} \delta^{i j} \frac{q^{2}}{q^{2}-c_{s}^{-2} \omega^{2}},
\end{aligned}
$$

defined in Sec. V, as well as the inverse compressibility $K^{-1}=-n_{0} m \frac{\omega^{2}}{q^{2}-c_{s}^{-2} \omega^{2}}$ (which agrees with the thermodynamic expression $K^{-1}=n_{0}^{2} \frac{\partial \mu}{\partial n_{0}}=n_{0} m c_{s}^{2}$ at $q=0$ ). In particular, the $\ell$-wave SF is indeed a superfluid-the even viscosity $\eta_{\mathrm{e}}$ vanishes to zeroth order in derivatives (see Ref. [3] for a subtlety in separating $K^{-1}$ from $\eta_{\mathrm{e}}$ ).

The $O(p)$ part of the Eq. (D1) is $P, T$-odd and vanishes when $s_{\theta}=0$. It is given by

$$
\begin{aligned}
\mathcal{L}_{\text {ind }, 1}= & -s_{\theta} n_{0} \omega_{t}+\frac{1}{2} \frac{s_{\theta} n_{0}}{m^{2} c_{s}^{2}} \frac{E^{i} \partial_{i} B}{\partial^{2}-c_{s}^{-2} \partial_{t}^{2}} \\
& -s_{\theta} n_{0} \frac{\left(E_{\omega}^{i}-\frac{1}{2 m} \partial_{i} B\right) \partial_{i} \sqrt{G}}{\partial^{2}-c_{s}^{-2} \partial_{t}^{2}} .
\end{aligned}
$$

The first and third lines produce the following odd viscosity [21],

$$
\begin{aligned}
& \eta_{\mathrm{o}}^{(1)}=-\frac{1}{2} s_{\theta} n_{0}, \\
& \eta_{\mathrm{o}}^{(2)}=\frac{1}{2} s_{\theta} n_{0} \frac{1}{q^{2}-c_{s}^{-2} \omega^{2}},
\end{aligned}
$$

and setting $\omega=0$ one obtains the leading terms in Eq. (18). By using the identity (up to a total derivative)

$$
E^{i} \partial_{i} B=\frac{1}{2} \varepsilon^{\mu \nu \rho} A_{\mu} \partial_{\nu} \partial^{2} A_{\rho}
$$

the second line of Eq. (D4) can be written as a nonlocal CS term,

$$
\mathcal{L}_{\text {ind }} \supset \frac{1}{2} \sigma_{\mathrm{o}}(\omega, q) \varepsilon^{\mu \nu \rho} A_{\mu} i p_{\nu} A_{\rho},
$$

with the odd (or Hall) conductivity $\sigma_{\mathrm{o}}(\omega, q)=$ $\sigma_{\mathrm{o}}^{0} q^{2} /\left(q^{2}-c_{s}^{-2} \omega^{2}\right), \quad \sigma_{\mathrm{o}}^{0}=s_{\theta} n_{0} / 2 m^{2} c_{s}^{2} \quad[21,40], \quad$ with $\sigma_{\mathrm{o}}(0, q)=\sigma_{\mathrm{o}}^{0}$ unquantized, and $\sigma_{\mathrm{o}}(\omega, 0)=0$, in accordance with the boundary $U(1)_{N}$-neutrality [25].

To demonstrate explicitly that $c$ cannot be extracted from the odd viscosity alone, it suffices to add the $O\left(p^{2}\right)$ term $\mathcal{L}_{2}^{(1)}=F_{1}(X) R \subset \mathcal{L}_{2}$. The situation is particularly simple for the special case $F_{1}(X)=-s_{\theta}^{2} P^{\prime}(X) / 4 m$. Then

$$
\begin{aligned}
P\left(X-\frac{s_{\theta}^{2}}{4 m} R\right) & =P(X)-\frac{s_{\theta}^{2}}{4 m} P^{\prime}(X) R+O\left(p^{4}\right) \\
& =P(X)+F_{1}(X) R+O\left(p^{4}\right),
\end{aligned}
$$

which shows that $F_{1}(X) R$ can be absorbed into $P(X)$ by a modification of $X$. The scalar $X-\frac{s_{\theta}^{2}}{4 m} R$ is useful because, unlike $X$, it depends on $A_{\mu}$ and $\omega_{\mu}$ only through the combination $\mathcal{A}_{\mu}=A_{\mu}+s_{\theta} \omega_{\mu}$. This is evident in Eq. (C1), where $B$ rather than $\mathcal{B}=B+\frac{s}{2} R$ appears. It is then clear that, to $O\left(p^{3}\right)$, adding $\mathcal{L}_{2}^{(1)}=F_{1}(X) R=-\frac{s_{\theta}^{2}}{4 m} P^{\prime}(X) R$ to $\mathcal{L}_{0}=P(X)$ amounts to changing $B$ to $\mathcal{B}$ in the induced 
Lagrangian Eq. (D1),

$$
\begin{aligned}
& \mathcal{L}_{\text {ind }}=P_{0} \sqrt{G}-n_{0} \mathcal{A}_{t} \\
& +\frac{1}{2} \frac{n_{0}}{m} \frac{\mathcal{B}^{2}-c_{s}^{-2} \mathcal{E}^{2}+\frac{s_{\theta} c_{s}^{-2}}{m} \mathcal{E}^{i} \partial_{i} \mathcal{B}-\frac{s_{\theta}^{2} c_{s}^{-2}}{4 m^{2}}(\partial \mathcal{B})^{2}}{\partial^{2}-c_{s}^{-2} \partial_{t}^{2}} \\
& -n_{0} \frac{m\left(\partial_{t} \sqrt{G}\right)^{2} / 2+\left(\mathcal{E}^{i}-\frac{s_{\theta}}{2 m} \partial_{i} \mathcal{B}\right) \partial_{i} \sqrt{G}}{\partial^{2}-c_{s}^{-2} \partial_{t}^{2}} .
\end{aligned}
$$

The only contribution to $\eta_{\mathrm{o}}$, beyond Eq. (D5), comes from the term proportional to $\mathcal{E}^{i} \partial_{i} \mathcal{B}$. By using the identity Eq. (D6) for
$\mathcal{A}_{\mu}$, this term can be written as the sum of nonlocal CS, WZ1, and WZ2 terms, which generalizes Eq. (D7) to

$$
\mathcal{L}_{\text {ind }} \supset \frac{1}{2} \sigma_{\mathrm{o}}(\omega, q) \varepsilon^{\mu \nu \rho}\left(A_{\mu}+s_{\theta} \omega_{\mu}\right) i p_{v}\left(A_{\rho}+s_{\theta} \omega_{\rho}\right) .
$$

Most importantly, this includes a nonlocal version of WZ2, which is indistinguishable from $\mathcal{L}_{\mathrm{gCS}}$ at $\omega=0$, where $\sigma_{\mathrm{o}}(0, q)=\sigma_{\mathrm{o}}^{0}$ is a constant. Noting that $F_{1}^{\prime}=-s_{\theta}^{2} P^{\prime \prime} / 4 m=$ $-\left(s_{\theta} / 2\right) \sigma_{\mathrm{o}}^{0}$, and comparing to Eq. (C8), it follows that $c$ and $F_{1}^{\prime}$ will enter the $\omega=0$ odd viscosity only through the combination $c+48 \pi s_{\theta} F_{1}^{\prime}$. In more detail, the odd viscosity tensor due to $\mathcal{L}_{0}+\mathcal{L}_{2}^{(1)}+\mathcal{L}_{\mathrm{gCS}}$, is given by

$$
\begin{aligned}
& \eta_{H}^{(1)}\left(\omega, q^{2}\right)=-\frac{1}{2} s_{\theta} n_{0}-\left(\frac{c}{24} \frac{1}{4 \pi}+\frac{s_{\theta}}{2} F_{1}^{\prime} \frac{q^{2}}{q^{2}-c_{s}^{-2} \omega^{2}}\right) q^{2}+O\left(q^{4}\right), \\
& \eta_{H}^{(2)}\left(\omega, q^{2}\right)=\frac{1}{2} s_{\theta} n_{0} \frac{1}{q^{2}-c_{s}^{-2} \omega^{2}}+\left(\frac{c}{24} \frac{1}{4 \pi}+\frac{s_{\theta}}{2} F_{1}^{\prime} \frac{q^{2}}{q^{2}-c_{s}^{-2} \omega^{2}}\right)+O\left(q^{2}\right),
\end{aligned}
$$

which, at $\omega=0$, is a special case of Eq. (12) of the main text.

Equation (D11) remains valid away from the special point $F_{1}=-s_{\theta}^{2} P^{\prime} / 4 m$, even though Eq. (D10) does not. Examining the perturbatively expanded $\mathcal{L}_{0}$ Eq. (C3) and $\mathcal{L}_{2}^{(1)}$ Eq. (C7), we see that a general $F_{1}$ amounts to replacing $B$ in Eq. (D1) with $B+\alpha \frac{s_{\theta}}{2} R$, where $\alpha=-\frac{4 m F_{1}^{\prime}}{s_{\theta}^{2} P^{\prime \prime}} \neq 1$ generically [as well as in the microscopic model Eq. (16)]. The general induced Lagrangian due to $\mathcal{L}_{0}+\mathcal{L}_{2}^{(1)}$, valid to $O\left(p^{3}\right)$, is then given by

$$
\begin{aligned}
\mathcal{L}_{\text {ind }}= & P_{0} \sqrt{G}-n_{0} \mathcal{A}_{t}+\frac{1}{2} \frac{n_{0}}{m} \frac{\mathcal{B}^{2}-c_{s}^{-2} \mathcal{E}^{2}+\frac{s_{\theta} c_{s}^{-2}}{m} \mathcal{E}^{i} \partial_{i}\left(B+\alpha \frac{s_{\theta}}{2} R\right)-\frac{s_{\theta}^{2} c_{s}^{-2}}{4 m^{2}}(\partial B)^{2}}{\partial^{2}-c_{s}^{-2} \partial_{t}^{2}} \\
& -n_{0} \frac{m\left(\partial_{t} \sqrt{G}\right)^{2} / 2+\left[\mathcal{E}^{i}-\frac{s_{\theta}}{2 m} \partial_{i}\left(B+\alpha \frac{s_{\theta}}{2} R\right)\right] \partial_{i} \sqrt{G}}{\partial^{2}-c_{s}^{-2} \partial_{t}^{2}}
\end{aligned}
$$

and, along with the $\mathcal{L}_{\mathrm{gCS}}$, produces the odd viscosity Eq. (D11). This expression does not depend on $A_{\mu}, \omega_{\mu}$ only through $\mathcal{A}_{\mu}$, but the terms contributing to Eq. (D11) still vanish $s_{\theta}=0$, which is why the improved odd viscosity due to Eq. (D12) vanishes. In addition to $\mathcal{L}_{2}^{(1)}$, the second order terms $\mathcal{L}_{2}^{(2)}, \mathcal{L}_{2}^{(3)}$ Eq. (C6) also produce $q^{2}$ corrections to the odd viscosity, but not to the improved odd viscosity.

Though Eq. (D10) describes only a part of $\mathcal{L}_{\text {ind }}$, and is nongeneric, it does reveal the analogy between CSFs and vars $=0$ QH states, described in the discussion Sec.VI in a very simple setting. Indeed, comparing Eq. (D10) with Eq. (12) we see that CSFs are analogous to vars $=0 \mathrm{QH}$ states, with $\bar{s}=-s_{\theta}=$ $-\ell / 2$, but with a nonlocal, nonquantized, Hall conductivity, in place of the filling factor $v / 2 \pi$. Additionally, both $\mathrm{QH}$ states and CSFs have the same gCS term Eq. (C8), with $c$ the boundary chiral central charge.

\section{APPENDIX E: DETAILED ANALYSIS OF THE MICROSCOPIC MODEL Eq. (13)}

\section{Symmetry}

The action $S_{\mathrm{m}}$ is invariant under $U(1)_{N}$ gauge transformations,

$$
\psi \mapsto e^{-i \alpha} \psi, \quad \Delta^{j} \mapsto e^{-2 i \alpha} \Delta^{j}, \quad A_{\mu} \mapsto A_{\mu}+\partial_{\mu} \alpha,
$$

which implies the current conservation $\partial_{\mu}\left(\sqrt{G} J^{\mu}\right)=0$, where $\sqrt{G} J^{\mu}=-\delta S / \delta A_{\mu}$. It is also clear that $S_{\mathrm{m}}$ is invariant under time-independent spatial diffeomorphisms, generated by $\delta x^{i}=\xi^{i}(\mathbf{x})$, if $\psi$ transforms as a function, $A_{\mu}$ as a 1 -form, $\Delta^{j}$ as a vector, and $G_{i j}$ as a rank-2 tensor. As described in Sec. II, due to its Galilean symmetry in flat space, $S_{\mathrm{m}}$ is also invariant under time-dependent spatial diffeomorphisms $\delta x^{i}=\xi^{i}(\mathbf{x}, t)$, provided one modifies the transformation rule of $A_{i}$ to Eq. (4).

\section{Effective action and fermionic Green's function}

Starting with the microscopic action Eq. (13), the effective action for the order parameter $\Delta$ in the $A, G$ background is obtained by integrating out the (generically) gapped fermion $\psi$,

$$
e^{i S_{\mathrm{eff}, \mathrm{m}}[\Delta ; A, G]}=\int \mathrm{D}\left(G^{1 / 4} \psi\right) \mathrm{D}\left(G^{1 / 4} \psi^{\dagger}\right) e^{i S_{\mathrm{m}}[\psi ; \Delta, A, G]},
$$

where $G^{1 / 4}=\left(\operatorname{det} G_{i j}\right)^{1 / 4}$ is the square root of the volume element $\sqrt{G}$. The form of the measure is fixed by the fact that the fundamental fermionic degree of freedom is the fermiondensity $\tilde{\psi}=G^{1 / 4} \psi$, which satisfies the usual canonical commutation relation $\left\{\tilde{\psi}^{\dagger}(\mathbf{x}), \tilde{\psi}(\mathbf{y})\right\}=\delta^{(2)}(\mathbf{x}-\mathbf{y})$ as an operator $[25,38,75,76]$. This is to be contrasted with $\left\{\psi^{\dagger}(\mathbf{x}), \psi(\mathbf{y})\right\}=$ $\delta^{(2)}(\mathbf{x}-\mathbf{y}) / \sqrt{G(\mathbf{x})}$ which ties the fermion to the background metric. 
In terms of $\tilde{\psi}$ the action Eq. (13) takes the form

$$
\begin{aligned}
S_{\mathrm{m}}= & \int d^{2} x d t\left[\tilde{\psi}^{\dagger} \frac{i}{2} \overleftrightarrow{\nabla}_{t} \tilde{\psi}-\frac{1}{2 m} G^{i j} \nabla_{i} \tilde{\psi}^{\dagger} \nabla_{j} \tilde{\psi}\right. \\
& \left.+\left(\frac{1}{2} \Delta^{i} \tilde{\psi}^{\dagger} \nabla_{i} \tilde{\psi}^{\dagger}+\text { h.c }\right)-\mathcal{U}\right],
\end{aligned}
$$

where $\nabla_{\mu}=\partial_{\mu}+i A_{\mu}-\frac{1}{4} \partial_{\mu} \log G$ is the covariant derivative for densities, and $\mathcal{U}=\frac{1}{2 \lambda} \sqrt{G} G_{i j} \Delta^{i *} \Delta^{j}$. Passing to the BdG form of the fermionic part of the action, in terms of the Nambu spinor-density $\tilde{\Psi}^{\dagger}=\left(\tilde{\psi}^{\dagger}, \tilde{\psi}\right)$ (which is a Majorana spinor-density [25]), one finds

$$
\begin{aligned}
S_{\mathrm{m}}= & \int d^{2} x d t\left\{\frac { 1 } { 2 } \tilde { \Psi } ^ { \dagger } \gamma ^ { 0 } \left[i \gamma^{0} \partial_{t}-A_{t}+\frac{1}{2 m} \nabla_{i} G^{i j} \nabla_{j}\right.\right. \\
& \left.\left.+\frac{i}{2} \gamma^{\tilde{A}}\left(e_{\tilde{A}}^{i} \partial_{i}+\partial_{i} e_{\tilde{A}}^{i}\right)\right] \tilde{\Psi}-\mathcal{U}\right\} \\
= & \int d^{2} x d t\left\{\frac{1}{2} \tilde{\Psi}^{\dagger} \gamma^{0} \mathcal{G}^{-1} \tilde{\Psi}-\mathcal{U}\right\},
\end{aligned}
$$

where derivatives act on all fields to the right; $\tilde{A}=1,2$ is an index for $U(1)_{N}$, viewed as a copy of $S O(2)$; the $\gamma$ matrices are $\gamma^{0}=\sigma^{z}, \gamma^{1}=-i \sigma^{x}, \gamma^{2}=i \sigma^{y}$, satisfying $\left\{\gamma^{\mu}, \gamma^{\nu}\right\}=$ $2 \eta^{\mu \nu}$ with $\eta^{\mu \nu}=\operatorname{diag}[1,-1,-1]$, and $\operatorname{tr}\left(\gamma^{0} \gamma^{1} \gamma^{2}\right)=2 i$; and

$$
e_{\tilde{A}}^{i}=\left(\begin{array}{ll}
\operatorname{Re} \Delta^{x} & \operatorname{Re} \Delta^{y} \\
\operatorname{Im} \Delta^{x} & \operatorname{Im} \Delta^{y}
\end{array}\right)
$$

is the emergent vielbein [25,35], to be distinguished from the background vielbein $E_{A}^{i}$ (with an $S O(2)_{L}$ index $A=1,2$ ) that appeared in the main text and that will be used momentarily. We also defined the inverse Green's function $\mathcal{G}^{-1}$. The effective action Eq. (E2) is then given by the logarithm of the Pfaffian

$$
\begin{aligned}
S_{\text {eff,m }} & =-i \log \operatorname{Pf}\left(i \gamma^{0} \mathcal{G}^{-1}\right)-\int d^{2} x d t \mathcal{U} \\
& =-\frac{i}{2} \log \operatorname{Det}\left(i \gamma^{0} \mathcal{G}^{-1}\right)-\int d^{2} x d t \mathcal{U}
\end{aligned}
$$

\section{Fermionic ground-state topology}

For a given $\Delta^{j}$, the fermion $\psi$ is gapped, unless the chemical potential $\mu$ or chirality $\ell=\operatorname{sgn}\left[\operatorname{Im}\left(\Delta^{x *} \Delta^{y}\right)\right]$ are tuned to 0 , and forms a fermionic topological phase characterized by the bulk Chern number. Assuming $A_{\mu}=0$ and space-time independent $\Delta^{i}, G^{i j}$, it is given by [13]

$$
C=\frac{1}{24 \pi^{2}} \operatorname{tr} \int d^{3} q \varepsilon^{\alpha \beta \gamma}\left(\mathcal{G} \partial_{\alpha} \mathcal{G}^{-1}\right)\left(\mathcal{G} \partial_{\beta} \mathcal{G}^{-1}\right)\left(\mathcal{G} \partial_{\gamma} \mathcal{G}^{-1}\right) \in \mathbb{Z},
$$

and determines the boundary chiral central charge $c=C / 2$ $[12,13,67,77]$. Here the fermionic Green's function $\mathcal{G}$ is Fourier transformed to Euclidian 3-momentum $q=\left(i q_{0}, \mathbf{q}\right)$ [see Eq. (E17)]. For the particular model Eq. (13) one finds

$$
c=-(\ell / 4)[\operatorname{sgn}(\mu)+\operatorname{sgn}(m)] \in\{0, \pm 1 / 2\},
$$

see Refs. $[12,13,25]$ for similar expressions. Note that the central charge is well defined for both $m>0$ and $m<0$, even though the single particle dispersion is not bounded from below in the latter, and many physical quantities naively diverge (we will see below that certain physical quantities diverge also with $m>0$ ). A negative mass can occur as an effective mass in lattice models, in which case the lattice spacing provides a natural cutoff [which must be smooth in momentum space for Eq. (E7) to hold]. In any case, a negative mass makes it possible to obtain both fundamental central charges $c= \pm 1 / 2$, for fixed $\ell$, within the model Eq. (13). All possible $c \in(1 / 2) \mathbb{Z}$ can then be obtained by stacking layers of the model Eq. (13) with the same $\ell$ but different $m, \mu$. Thus, the model Eq. (13) suffices to generate a representative for all topological phases of the $p$-wave CSF. For concreteness, below we will work only with $m>0$, in which case $c$ is given by Eq. (14).

\section{Symmetry breaking and bosonic ground state in the presence of a background metric}

For time independent fields $A, G, \Delta$ the effective action reduces to

$$
S_{\mathrm{eff}, \mathrm{m}}[\Delta ; G]=-\int d^{2} x d t \varepsilon_{0}[\Delta ; G],
$$

where $\varepsilon_{0}$ is the ground-state energy-density as a function of the fields. In flat space $G_{i j}=\delta_{i j}$, with $A_{t}=-\mu$ and $A_{i}=0$, and assuming $\Delta$ is constant, it is given by $[13,25]$

$$
\varepsilon_{0}=\frac{1}{2} \int \frac{d^{2} \mathbf{q}}{(2 \pi)^{2}}\left(\xi_{\mathbf{q}}-\sqrt{\xi_{\mathbf{q}}^{2}+g^{i j} q_{i} q_{j}}\right)+\frac{1}{2 \lambda} \delta_{i j} g^{i j},
$$

where

$$
\xi_{\mathbf{q}}=|\mathbf{q}|^{2} / 2 m-\mu
$$

is the single particle dispersion, and $g^{i j}=\Delta^{(i} \Delta^{j) *}=\delta^{\tilde{A} \tilde{B}} e_{\tilde{A}}^{i} e_{\tilde{B}}^{j}$ is the emergent metric - a dynamical metric to be distinguished from the background metric $G^{i j}$. The ground-state configuration of $g^{i j}$ is determined by minimizing $\varepsilon_{0}$, while the overall phase $\theta$ of the order parameter and the chirality $\ell$, of which $g^{i j}$ is independent, are left undetermined. Thus, $g^{i j}$ corresponds to a massive Higgs field, while $\theta$ is a Goldstone field. The energy-density Eq. (E10) is UV divergent, and requires regularization. We do this in the simplest manner, by introducing a momentum cutoff $q^{2}<\Lambda^{2}$. Since the divergence disappears for $g^{i j}=0$ (assuming $m>0$ ), this can be thought of as a small, but nonvanishing, range $1 / \Lambda$ for the interaction mediated by $\Delta$. With a finite $\Lambda$, the energy density is well defined and has a unique global minimum at $g^{i j}=$ $\Delta_{0}^{2} \delta^{i j}$, with $\Delta_{0}$ determined by the self-consistent equation

$$
\frac{1}{4} \int^{\Lambda} \frac{d^{2} \boldsymbol{q}}{(2 \pi)^{2}} \frac{|\boldsymbol{q}|^{2}}{\sqrt{\xi_{\boldsymbol{q}}^{2}+\Delta_{0}^{2}|\boldsymbol{q}|^{2}}}=\frac{1}{\lambda} .
$$

For $\mu>0$ the noninteracting system has a Fermi surface, and a solution exists for all $\lambda>0$, which is the statement of the BCS instability. For $\mu<0$, the noninteracting system is gapped, and a solution exists if the interaction is large enough compared with the gap, $\lambda \Lambda^{-4} \gtrsim|\mu|$.

Consider now the case of a general constant metric $G_{i j}$, and let us introduce a constant vielbein $E$ such that $G_{i j}=$ $E_{i}^{A} \delta_{A B} E_{j}^{B}$. The inverse transpose $E^{-T}=\left(E^{-1}\right)^{T}$ is given in coordinates by $E_{A}^{i}$. We also introduce the internal order pa- 
rameter $\Delta^{A}=E_{i}^{A} \Delta^{i}$. The action Eq. (E3) then reduces to

$$
\begin{aligned}
S_{\mathrm{m}}= & \int d^{2} x d t\left[\tilde{\psi}^{\dagger} i \partial_{t} \tilde{\psi}-\frac{\delta^{A B}}{2 m} E_{A}^{i} \partial_{i} \tilde{\psi}^{\dagger} E_{B}^{j} \partial_{j} \tilde{\psi}\right. \\
& \left.+\left(\frac{1}{2} \Delta^{A} E_{A}^{i} \tilde{\psi}^{\dagger} \partial_{i} \tilde{\psi}^{\dagger}+\text { H.c. }\right)-\frac{1}{2 \lambda} \delta_{A B} \Delta^{A *} \Delta^{B}\right] .
\end{aligned}
$$

This is identical to the flat space case, with $\partial_{i}$ replaced by $E_{A}^{i} \partial_{i}$. We also need to change the UV cutoff to $\delta^{A B} E_{A}^{i} q_{i} E_{B}^{j} q_{j}=$ $G^{i j} q_{i} q_{j}<\Lambda^{2}$. This in natural since we interpret $\Lambda^{2}$ as a range of the interaction mediated by $\Delta$, which should be defined in terms of the geodesic distance rather than the Euclidian distance. It follows that the flat space result Eq. (E10) is modified to

$$
\begin{aligned}
\varepsilon_{0}= & \frac{1}{2} \int_{\left|E^{-T} \mathbf{q}\right|^{2}<\Lambda^{2}} \frac{d^{2} \mathbf{q}}{(2 \pi)^{2}}\left(\xi_{E^{-T} \mathbf{q}}-\sqrt{\xi_{E^{-T} \mathbf{q}}^{2}+g^{A B} E_{A}^{i} E_{B}^{j} q_{i} q_{j}}\right) \\
& +\frac{1}{2 \lambda} \delta_{A B} g^{A B} \\
= & \frac{1}{2} \sqrt{G} \int_{q^{2}<\Lambda^{2}} \frac{d^{2} \mathbf{k}}{(2 \pi)^{2}}\left(\xi_{\mathbf{k}}-\sqrt{\xi_{\mathbf{k}}^{2}+g^{A B} k_{A} k_{B}}\right) \\
& +\frac{1}{2 \lambda} \delta_{A B} g^{A B},
\end{aligned}
$$

where $\mathbf{k}=E^{-T} \mathbf{q}$, or $k_{A}=E_{A}^{i} q_{i}$, and $g^{A B}=\Delta^{(A} \Delta^{B) *}=$ $\delta^{\tilde{A} \tilde{B}} e_{\tilde{A}}^{A} e_{\tilde{B}}^{B}$ is the internal emergent metric. This is identical to the $G_{i j}=\delta_{i j}$ result Eq. (E10), apart from the volume element $\sqrt{G}$, and the fact that it is the internal metric $g^{A B}$ that appears, rather than $g^{i j}$. It is then clear that minimizing Eq. (E14) with respect to $g^{A B}$ gives

$$
g^{A B}=\Delta_{0}^{2} \delta^{A B}, \quad \text { or }{ }^{i j}=\Delta_{0}^{2} G^{i j},
$$

with the same $\Delta_{0}$ of Eq. (E12), which is $G$ independent. Thus, the emergent metric is proportional to the background metric in the ground state. This solution corresponds to emergent vielbeins $e_{\tilde{A}}^{A} \in O(2)$, or order parameters $\Delta^{A}=\Delta_{0} e^{2 i \theta}(1, \pm i)$, which is the $p_{x} \pm i p_{y}$ configuration, and implies the SSB pattern

$$
\begin{aligned}
& \left(\mathbb{Z}_{2, T} \ltimes U(1)_{N}\right) \times\left(\mathbb{Z}_{2, P} \ltimes S O(2)_{L}\right) \\
& \rightarrow \begin{cases}\mathbb{Z}_{2, P T} \ltimes U(1)_{L-\frac{\ell}{2} N} & \ell \in 2 \mathbb{Z}+1, \\
\mathbb{Z}_{2, P T} \ltimes U(1)_{L-\frac{\ell}{2} N} \times \mathbb{Z}_{2,(-1)^{N}} & \ell \in 2 \mathbb{Z}\end{cases}
\end{aligned}
$$

described less formally in the main text. Note that fermion parity $\mathbb{Z}_{2,(-1)^{N}}$ is the $\mathbb{Z}_{2}$ subgroup of $U(1)_{L-\frac{\ell}{2} N}$ for odd $\ell$. For $\Delta^{j}$, we find the ground-state configuration Eq. (15) - a result that was stated previously in the literature $[12,21,23,66,68]$ and is derived here to zeroth order in derivatives.

As described in Appendix E, we will ignore the massive Higgs fluctuations, and obtain $S_{\text {eff }}[\theta ; A, G]$ by plugging the ground-state configuration Eq. (15) into the functional Pfaffian (E6).

\section{Perturbative expansion}

We now write $E_{A}^{i}=\delta_{A}^{i}+H_{A}^{i}$ and $e_{\tilde{A}}^{A}=\Delta_{0} \delta_{\tilde{A}}^{A}$ [which corresponds to $\left.\Delta^{A}=\Delta_{0}(1, i)^{A}\right]$ and expand Eq. (E4) to second order in $H, A$. Due to $S O(2)_{L}$ gauge symmetry, the antisymmetric part of $H$ can be interpreted as the Goldstone field, $\theta=\left(s_{\theta} / 2\right) \varepsilon_{A B} H^{A B}$, as explained in Appendix D. The $p_{x}-i p_{y}$ configuration $\Delta^{A}=\Delta_{0}(1,-i)^{A}$ can be incorporated by changing the sign of one of the gamma matrices $\gamma^{\tilde{A}}$. The expansion in $H, A$ produces a splitting of the propagator into an unperturbed propagator and vertices, $\mathcal{G}^{-1}=\mathcal{G}_{0}^{-1}+\mathcal{V}$, where $\mathcal{V}$ further splits as $\mathcal{V}=\mathcal{V}_{1}+\mathcal{V}_{2}$, where $\mathcal{V}_{1}\left(\mathcal{V}_{2}\right)$ is first (second) order in the fields. The terms in $\mathcal{V}_{2}$ are often referred to as contact terms. Using Eq. (A1) we find the explicit form of $\mathcal{G}_{0}^{-1}, \mathcal{V}_{1}, \mathcal{V}_{2}$ in Fourier components,

$$
\begin{aligned}
\mathcal{G}_{0}^{-1}(q)= & -\gamma^{0} q_{0}-\Delta_{0} \gamma^{j} q_{j}-\xi_{\mathbf{q}}, \\
\mathcal{V}_{1}(q, p)= & -A_{t, p}-\Delta_{0} \gamma^{A}\left(H_{A}^{i}\right)_{p} q_{i} \\
& -\frac{1}{m}\left[q_{i} q_{j}-\frac{1}{4}\left(p_{i} p_{j}-\delta_{i j} p^{2}\right)\right] H_{p}^{i j}+\gamma^{0} \frac{1}{m} A_{p}^{j} q_{j}, \\
\mathcal{V}_{2}(q, 0)= & -\frac{1}{2 m}\left(H_{A}^{i} H^{A j}\right)_{p=0} q_{i} q_{j}-\frac{1}{8 m}\left(\partial^{j} H_{A}^{A} \partial_{j} H_{B}^{B}\right)_{p=0} \\
& -\gamma^{0} \frac{2}{m}\left(A_{i} H^{(i j)}\right)_{p=0} q_{j}-\frac{1}{2 m}\left(A^{j} A_{j}\right)_{p=0} . \quad \text { (E17) }
\end{aligned}
$$

Here $(\cdots)_{p}$ denotes the $p$ Fourier component of the field $(\cdots)$, and we set $p=0$ in $\mathcal{V}_{2}$ since only this component will be relevant. The unperturbed Greens's function is given explicitly by

$$
\mathcal{G}_{0}(q)=-\frac{q_{0} \gamma^{0}+\Delta_{0} q_{i} \gamma^{i}-\xi_{\mathbf{q}}}{q_{0}^{2}-q_{i} q^{i}-\xi_{\mathbf{q}}^{2}} .
$$

The perturbative expansion of $S_{\text {eff }}$ is obtained from Eq. (E6) by using $\log [\operatorname{Det}(\cdot)]=\operatorname{Tr}[\log (\cdot)]$, and expanding the logarithm in $\mathcal{V}$,

$$
\begin{aligned}
S_{\text {eff,m }}= & -i \operatorname{Tr}\left\{\log \left[i \gamma^{0}\left(\mathcal{G}_{0}^{-1}+\mathcal{V}\right)\right]\right\} \\
= & -\frac{i}{2} \operatorname{Tr}\left(\log i \gamma^{0} \mathcal{G}_{0}^{-1}\right)-\frac{i}{2} \operatorname{Tr}\left(\mathcal{G}_{0} \mathcal{V}\right) \\
& +\frac{i}{4} \operatorname{Tr}\left(\mathcal{G}_{0} \mathcal{V}\right)^{2}+O\left(\mathcal{V}^{3}\right) \\
= & -\frac{i}{2} \operatorname{Tr}\left(\mathcal{G}_{0} \mathcal{V}_{1}\right)-\frac{i}{2} \operatorname{Tr}\left(\mathcal{G}_{0} \mathcal{V}_{2}\right) \\
& +\frac{i}{4} \operatorname{Tr}\left(\mathcal{G}_{0} \mathcal{V}_{1} \mathcal{G}_{0} \mathcal{V}_{1}\right)+\cdots,
\end{aligned}
$$

where in the last line we kept explicit only terms at first and second order in $H, A$ (the term of zeroth order was described in the previous section). Writing the functional traces as integrals over Fourier components and traces over spinor indices, we then find

$$
\begin{aligned}
S_{\mathrm{eff}, \mathrm{m}}= & -\frac{i}{2} \operatorname{tr} \int_{q} \mathcal{V}_{1}(q, 0) \mathcal{G}_{0}(q)-\frac{i}{2} \operatorname{tr} \int_{q} \mathcal{V}_{2}(q, 0) \mathcal{G}_{0}(q) \\
& +\frac{i}{4} \operatorname{tr} \int_{p, q} \mathcal{G}_{0}\left(q-\frac{1}{2} p\right) \mathcal{V}_{1}(q,-p) \mathcal{G}_{0} \\
& \times\left(q+\frac{1}{2} p\right) \mathcal{V}_{1}(q, p)+\cdots,
\end{aligned}
$$

where $\int_{q}=\int \frac{d^{2} q d q_{0}}{(2 \pi)^{3}}$. We are interested in $S_{\text {eff }}$ to third order in derivatives, which amounts to expanding the above expres- 
sion to $O\left(p^{3}\right)$, and evaluating the resulting traces and integrals. These computations are performed in the accompanying Mathematica notebook [78].

The result, focusing on terms relevant for $\eta_{\mathrm{o}}, \tilde{\eta}_{\mathrm{o}}$ to $O\left(q^{2}\right)$, is compatible with the general effective action of Sec. III and Appendix C, as confirmed by comparing Eq. (E20) to the perturbatively expanded $S_{\text {eff }}$. This comparison provides explicit expressions for all of the coefficients that appear in $S_{\text {eff }}$, as we now describe. The ground-state pressure $P(\mu)$ diverges logarithmically, and is given by

$$
\begin{aligned}
P & =\frac{1}{2} \int^{\Lambda} \frac{d^{2} q}{(2 \pi)^{2}}\left[\frac{q^{2}}{2 m}-\frac{\frac{1}{2} \Delta_{0}^{2} q^{2}+\frac{q^{2}}{2 m}\left(\frac{q^{2}}{2 m}-\mu\right)}{\sqrt{\Delta_{0}^{2} q^{2}+\left(\frac{q^{2}}{2 m}-\mu\right)^{2}}}\right] \\
& =-\frac{m^{3} \Delta_{0}^{4}}{4 \pi}\left(1-2 \frac{\mu}{m \Delta_{0}^{2}}\right) \log \Lambda+O\left(\Lambda^{0}\right) .
\end{aligned}
$$

Directly computing the ground-state density $n_{0}$ and leading odd viscosity $\eta_{\mathrm{o}}^{(1)}$ one finds

$$
\begin{aligned}
n_{0} & =\frac{1}{2} \int \frac{d^{2} q}{(2 \pi)^{2}}\left[1-\frac{\left(\frac{q^{2}}{2 m}-\mu\right)}{\sqrt{\Delta_{0}^{2} q^{2}+\left(\frac{q^{2}}{2 m}-\mu\right)^{2}}}\right] \\
& =\frac{m^{2} \Delta_{0}^{2}}{2 \pi} \log \Lambda+O\left(\Lambda^{0}\right), \\
\eta_{0}^{(1)} & =-\frac{\ell}{16} \int \frac{d^{2} q}{(2 \pi)^{2}} \frac{\Delta_{0}^{2} q^{2}\left(\frac{q^{2}}{2 m}+\mu\right)}{\left[\left(\frac{q^{2}}{2 m}-\mu\right)^{2}+q^{2} \Delta_{0}^{2}\right]^{3 / 2}} \\
& =-\frac{\ell m^{2} \Delta_{0}^{2}}{8 \pi} \log \Lambda+O\left(\Lambda^{0}\right),
\end{aligned}
$$

so the relations $n_{0}=P^{\prime}(\mu)$, and $\eta_{0}^{(1)}=-(\ell / 4) n_{0}$, described in the main text, are maintained to leading order in the cutoff.

As explained in Appendix E4, the cutoff $\Lambda$ corresponds to a nonvanishing interaction range, which softens the contact interaction in the model Eq. (13). With a space-independent metric, a smooth cutoff can easily be implemented by replacing

$$
\Delta^{A} E_{A}^{j} \tilde{\psi}_{-\mathbf{q}}^{\dagger} i q_{j} \tilde{\psi}_{\mathbf{q}}^{\dagger} \mapsto \Delta^{A} E_{A}^{j} \tilde{\psi}_{-\mathbf{q}}^{\dagger}\left(i q_{j} e^{-q_{k} q_{l} G^{k l} / \Lambda^{2}}\right) \tilde{\psi}_{\mathbf{q}}^{\dagger},
$$

for example, in the Fourier transformed Eq. (E13), and should lead to the exact relations $n_{0}=P^{\prime}(\mu), \eta_{\mathrm{o}}^{(1)}=-(\ell / 4) n_{0}$. However, a computation of the $q^{2}$ correction to $\eta_{\mathrm{o}}$ requires a spacedependent metric, where a nonvanishing interaction range involves the geodesic distance and complicates the vertex $\mathcal{V}$ in Eq. (E17) considerably. Moreover, all other coefficients in $S_{\text {eff }}$ converge, and we can therefore work with the simple contact interaction, $\Lambda=\infty$.

The coefficients $P^{\prime \prime}, F_{1}^{\prime}, F_{2}, F_{3}$ were presented in Appendix E. The remaining coefficients $F_{4}, F_{5}, F_{6}$ are irrelevant for the quantities discussed in the main text and are presented here for completeness,

$$
\begin{aligned}
& F_{4}=\frac{1}{24 \pi \mu}\left\{\begin{array}{l}
\frac{\kappa-2}{2} \\
\frac{1}{1+2 \kappa}
\end{array}, \quad F_{5}=\frac{1}{24 \pi \mu \Delta_{0}^{2}}\left\{\begin{array}{l}
1 \\
-\frac{1}{(1+2 \kappa)^{2}}
\end{array},\right.\right. \\
& F_{6}=-\frac{\kappa}{24 \pi \mu}\left\{\begin{array}{l}
\frac{1}{2} \frac{1}{(1+2 \kappa)^{2}} .
\end{array}\right.
\end{aligned}
$$

As stated in Appendix E, there is a sense in which the relativistic limit $\kappa \rightarrow 0$, or $m \rightarrow \infty$ reproduces the effective action of a massive Majorana spinor in RiemannCartan space-time [25,69]. In particular, in the limit $\kappa \rightarrow 0$ the dimensionless coefficients Eq. (16) are all quantized, as follows from dimensional analysis. Apart from $c$, only the coefficient $F_{1}^{\prime}$ is discontinuous at $\mu=0$ within this limit, with a quantized discontinuity $-(\ell / 4)\left[F_{1}^{\prime}\left(0^{+}\right)-F_{1}^{\prime}\left(0^{-}\right)\right]=$ $(\ell / 2) / 96 \pi$ that matches the coefficient $\beta$ of the gravitational pseudo Chern-Simons term of Ref. [25]. As anticipated in Ref. [25], the coefficient $c$ remains quantized away from the relativistic limit, while $F_{1}^{\prime}$ does not. Taking the relativistic limit of the dimensionful coefficients Eq. (E25), one finds $F_{6}=0$, while $F_{4}=-\Delta_{0}^{2} F_{5} \neq 0$ describe a relativistic term which is second order in torsion, and was not written explicitly in Refs. [25,69].

Finally, we note that our perturbative computation of the gCS term is analogous to the computations of Refs. [79-83] for relativistic fermions and reduces to these as $\kappa \rightarrow 0$.
[1] J. E. Avron, R. Seiler, and P. G. Zograf, Phys. Rev. Lett. 75, 697 (1995).

[2] J. E. Avron, J. Stat. Phys. 92, 543 (1998).

[3] B. Bradlyn, M. Goldstein, and N. Read, Phys. Rev. B 86, 245309 (2012).

[4] C. Hoyos, Int. J. Mod. Phys. B 28, 1430007 (2014).

[5] M. F. Lapa and T. L. Hughes, Phys. Rev. E 89, 043019 (2014).

[6] A. Lucas and P. Surówka, Phys. Rev. E 90, 063005 (2014); M. Sherafati, A. Principi, and G. Vignale, Phys. Rev. B 94, 125427 (2016); L. V. Delacrétaz and A. Gromov, Phys. Rev. Lett. 119, 226602 (2017); T. Scaffidi, N. Nandi, B. Schmidt, A. P. Mackenzie, and J. E. Moore, ibid. 118, 226601 (2017); T. I. Tuegel and T. L. Hughes, Phys. Rev. B 96, 174524 (2017); S. Ganeshan and A. G. Abanov, Phys. Rev. Fluids 2, 094101 (2017); D. Banerjee, A. Souslov, A. G. Abanov, and
V. Vitelli, Nat. Commun. 8, 1573 (2017); A. Bogatskiy and P. Wiegmann, Phys. Rev. Lett. 122, 214505 (2019); T. Holder, R. Queiroz, and A. Stern, ibid. 123, 106801 (2019); A. Souslov, K. Dasbiswas, M. Fruchart, S. Vaikuntanathan, and V. Vitelli, ibid. 122, 128001 (2019).

[7] V. Soni, E. Bililign, S. Magkiriadou, S. Sacanna, D. Bartolo, M. J. Shelley, and W. T. M. Irvine, arXiv:1812.09990.

[8] A. I. Berdyugin, S. G. Xu, F. M. D. Pellegrino, R. Krishna Kumar, A. Principi, I. Torre, M. Ben Shalom, T. Taniguchi, K. Watanabe, I. V. Grigorieva, M. Polini, A. K. Geim, and D. A. Bandurin, Science 364, 162 (2019).

[9] D. Thouless, M. Kohmoto, M. Nightingale, and M. Den Nijs, Phys. Rev. Lett. 49, 405 (1982); J. Avron, R. Seiler, and B. Simon, ibid. 51, 51 (1983); M. F. Golterman, K. Jansen, and D. B. Kaplan, Phys. Lett. B 301, 219 (1993); X.-L. Qi, T. L. Hughes, and S.-C. Zhang, Phys. Rev. B 78, 195424 (2010); 
"Scientifc background on the Nobel Prize in physics 2016" (2016); B. Mera, arXiv:1705.04394.

[10] N. Read, Phys. Rev. B 79, 045308 (2009).

[11] N. Read and E. Rezayi, Phys. Rev. B 84, 085316 (2011).

[12] N. Read and D. Green, Phys. Rev. B 61, 10267 (2000).

[13] G. Volovik, The Universe in a Helium Droplet (Oxford University Press, New York, 2009).

[14] D. Vollhardt and P. Wölfle, The Superfluid Phases of Helium 3 (Courier Corporation, North Chelmsford, MA, 2013).

[15] L. V. Levitin, R. G. Bennett, A. Casey, B. Cowan, J. Saunders, D. Drung, T. Schurig, and J. M. Parpia, Science 340, 841 (2013); P. M. Walmsley and A. I. Golov, Phys. Rev. Lett. 109, 215301 (2012); H. Ikegami, Y. Tsutsumi, and K. Kono, Science 341, 59 (2013); N. Zhelev, T. S. Abhilash, E. N. Smith, R. G. Bennett, X. Rojas, L. Levitin, J. Saunders, and J. M. Parpia, Nat. Commun. 8, 15963 (2017).

[16] C. Zhang, S. Tewari, R. M. Lutchyn, and S. Das Sarma, Phys. Rev. Lett. 101, 160401 (2008); M. Sato, Y. Takahashi, and S. Fujimoto, ibid. 103, 020401 (2009); G. Liu, N. Hao, S.-L. Zhu, and W. M. Liu, Phys. Rev. A 86, 013639 (2012); N. Hao, G. Liu, N. Wu, J. Hu, and Y. Wang, ibid. 87, 053609 (2013); S.-L. Zhang, L.-J. Lang, and Q. Zhou, Phys. Rev. Lett. 115, 225301 (2015); A. Boudjemâa, Phys. Lett. A 381, 1745 (2017); N. Hao, H. Guo, and P. Zhang, New J. Phys. 19, 083020 (2017).

[17] C. Kallin and J. Berlinsky, Rep. Prog. Phys. 79, 054502 (2016); M. Sato and Y. Ando, ibid. 80, 076501 (2017).

[18] J.-P. Xu, M.-X. Wang, Z. L. Liu, J.-F. Ge, X. Yang, C. Liu, Z. A. Xu, D. Guan, C. L. Gao, D. Qian, Y. Liu, Q.-H. Wang, F.-C. Zhang, Q.-K. Xue, and J.-F. Jia, Phys. Rev. Lett. 114, 017001 (2015); G. C. Ménard, S. Guissart, C. Brun, R. T. Leriche, M. Trif, F. Debontridder, D. Demaille, D. Roditchev, P. Simon, and T. Cren, Nat. Commun. 8, 2040 (2017).

[19] C. Wang, A. Vishwanath, and B. I. Halperin, Phys. Rev. B 98, 045112 (2018); D. F. Mross, Y. Oreg, A. Stern, G. Margalit, and M. Heiblum, Phys. Rev. Lett. 121, 026801 (2018); S. H. Simon, Phys. Rev. B 97, 121406 (2018); D. E. Feldman, ibid. 98, 167401 (2018); K. K. W. Ma and D. E. Feldman, ibid. 99, 085309 (2019).

[20] D. T. Son, Phys. Rev. X 5, 031027 (2015); Annu. Rev. Condens. Matter Phys. 9, 397 (2018).

[21] C. Hoyos, S. Moroz, and D. T. Son, Phys. Rev. B 89, 174507 (2014)

[22] A. Shitade and T. Kimura, Phys. Rev. B 90, 134510 (2014).

[23] S. Moroz and C. Hoyos, Phys. Rev. B 91, 064508 (2015).

[24] P. N. Brusov and V. N. Popov, Phys. Lett. A 87, 472 (1982); G. E. Volovik and M. A. Zubkov, J. Low Temp. Phys. 175, 486 (2013); J. A. Sauls, Phys. Rev. B 95, 094515 (2017); W.-H. Hsiao, 100, 094510 (2019).

[25] O. Golan and A. Stern, Phys. Rev. B 98, 064503 (2018).

[26] As can be seen by considering a mixture of CSFs with different $\ell$ s, where $N$ and $L$ are completely broken, and $s \equiv-2 \eta_{\mathrm{o}}^{(1)} / n=$ $\sum_{i} n_{i}\left(\ell_{i} / 2\right) / \sum_{i} n_{i}$ is no longer quantized.

[27] L. Alvarez-Gaume and E. Witten, Nucl. Phys. B 234, 269 (1984); R. A. Bertlmann, Anomalies in Quantum Field Theory, Vol. 91 (Oxford University Press, Oxford, 2000); F. Bastianelli and P. Van Nieuwenhuizen, Path Integrals and Anomalies in Curved Space (Cambridge University Press, Cambridge, 2006).

[28] C. Kane and M. P. Fisher, Phys. Rev. B 55, 15832 (1997).

[29] A. Cappelli, M. Huerta, and G. R. Zemba, Nucl. Phys. B 636, 568 (2002).
[30] S. Jezouin, F. Parmentier, A. Anthore, U. Gennser, A. Cavanna, Y. Jin, and F. Pierre, Science 342, 601 (2013); M. Banerjee, M. Heiblum, A. Rosenblatt, Y. Oreg, D. E. Feldman, A. Stern, and V. Umansky, Nature 545, 75 (2017); M. Banerjee, M. Heiblum, V. Umansky, D. E. Feldman, Y. Oreg, and A. Stern, ibid. 559, 205 (2018); Y. Kasahara, T. Ohnishi, Y. Mizukami, O. Tanaka, S. Ma, K. Sugii, N. Kurita, H. Tanaka, J. Nasu, Y. Motome, T. Shibauchi, and Y. Matsuda, ibid. 559, 227 (2018).

[31] P. Kraus and F. Larsen, J. High Energy Phys. 01 (2006) 022.

[32] S. Ryu, J. E. Moore, and A. W. Ludwig, Phys. Rev. B 85, 045104 (2012).

[33] M. Stone, Phys. Rev. B 85, 184503 (2012).

[34] E. Witten, Rev. Mod. Phys. 88, 035001 (2016).

[35] G. E. Volovik, Pis'ma Zh. Eksp. Teor. Fiz. 51, 111 (1990) [JETP Lett. 51, 125 (1990)]

[36] Z. Wang, X.-L. Qi, and S.-C. Zhang, Phys. Rev. B 84, 014527 (2011).

[37] B. Bradlyn and N. Read, Phys. Rev. B 91, 165306 (2015).

[38] A. G. Abanov and A. Gromov, Phys. Rev. B 90, 014435 (2014).

[39] S. Klevtsov and P. Wiegmann, Phys. Rev. Lett. 115, 086801 (2015).

[40] G. Volovik, Phys. Lett. A 128, 277 (1988); J. Goryo and K. Ishikawa, ibid. 246, 549 (1998); 260, 294 (1999); A. Furusaki, M. Matsumoto, and M. Sigrist, Phys. Rev. B 64, 054514 (2001); M. Stone and R. Roy, ibid. 69, 184511 (2004); R. Roy and C. Kallin, ibid. 77, 174513 (2008); R. M. Lutchyn, P. Nagornykh, and V. M. Yakovenko, ibid. 77, 144516 (2008).

[41] D. Ariad, E. Grosfeld, and B. Seradjeh, Phys. Rev. B 92, 035136 (2015).

[42] D. Son and M. Wingate, Ann. Phys. 321, 197 (2006).

[43] T. Qin, Q. Niu, and J. Shi, Phys. Rev. Lett. 107, 236601 (2011); A. Shitade, Prog. Theor. Exp. Phys. 2014, 123 I01 (2014); A. Gromov and A. G. Abanov, Phys. Rev. Lett. 114, 016802 (2015); R. Nakai, S. Ryu, and K. Nomura, New J. Phys. 18, 023038 (2016); Phys. Rev. B 95, 165405 (2017); A. Kapustin and L. Spodyneiko, arXiv:1905.06488.

[44] B. Bradlyn and N. Read, Phys. Rev. B 91, 125303 (2015).

[45] C. Hoyos and D. T. Son, Phys. Rev. Lett. 108, 066805 (2012).

[46] A. Gromov and A. G. Abanov, Phys. Rev. Lett. 113, 266802 (2014).

[47] O. Andreev, Phys. Rev. D 89, 064012 (2014).

[48] M. Geracie, D. T. Son, C. Wu, and S.-F. Wu, Phys. Rev. D 91, 045030 (2015).

[49] O. Andreev, Phys. Rev. D 91, 024035 (2015).

[50] M. Geracie, Phys. Rev. B 95, 134510 (2017).

[51] S. M. Carroll, Spacetime and Geometry: An Introduction to General Relativity, Vol. 1 (Cambridge University Press, London, UK, 2004).

[52] S.-S. Chern and J. Simons, Ann. Math. 99, 48 (1974).

[53] R. Jackiw and S.-Y. Pi, Phys. Rev. D 68, 104012 (2003).

[54] E. Witten, arXiv:0706.3359.

[55] R. F. Pérez, Class. Quantum Grav. 27, 135015 (2010).

[56] A. Gromov, K. Jensen, and A. G. Abanov, Phys. Rev. Lett. 116, 126802 (2016).

[57] F. Ferrari and S. Klevtsov, J. High Energy Phys. 12 (2014) 86.

[58] T. Can, M. Laskin, and P. Wiegmann, Phys. Rev. Lett. 113, 046803 (2014). 
[59] A. Gromov, G. Y. Cho, Y. You, A. G. Abanov, and E. Fradkin, Phys. Rev. Lett. 114, 016805 (2015).

[60] T. Can, M. Laskin, and P. B. Wiegmann, Ann. Phys. 362, 752 (2015).

[61] S. Klevtsov, X. Ma, G. Marinescu, and P. Wiegmann, Commun. Math. Phys. 349, 819 (2016).

[62] S. Klevtsov, arXiv:1712.09980.

[63] A. Cappelli and L. Maffi, J. Phys. A: Math. Theor. 51, 365401 (2018).

[64] These need to be modified as in Refs. [45,47,49] for LGS.

[65] G. E. Volovik, Soviet Physics-JETP (English Translation), 67, 1804 (1988).

[66] A. Quelle, C. M. Smith, T. Kvorning, and T. H. Hansson, Phys. Rev. B 94, 125137 (2016).

[67] S. Ryu, A. P. Schnyder, A. Furusaki, and A. W. Ludwig, New J. Phys. 12, 065010 (2010).

[68] S. Moroz, C. Hoyos, and L. Radzihovsky, Phys. Rev. B 93, 024521 (2016).

[69] T. L. Hughes, R. G. Leigh, and O. Parrikar, Phys. Rev. D 88, 025040 (2013).
[70] J. Hoeller and N. Read, Bull. Am. Phys. Soc. B07.009 (2018).

[71] F. Peña Benitez, K. Saha, and P. Surówka, Phys. Rev. B 99, 045141 (2019).

[72] B. Offertaler and B. Bradlyn, Phys. Rev. B 99, 035427 (2019).

[73] F. Rose, O. Golan, and S. Moroz (unpublished).

[74] T. L. Hughes, R. G. Leigh, and E. Fradkin, Phys. Rev. Lett. 107, 075502 (2011).

[75] S. W. Hawking, Commun. Math. Phys. 55, 133 (1977).

[76] K. Fujikawa, Phys. Rev. Lett. 44, 1733 (1980).

[77] A. Kitaev, Ann. Phys. 321, 2 (2006).

[78] See Supplemental Material at http://link.aps.org/supplemental/ 10.1103/PhysRevB.100.104512 for detailed symbolic computations.

[79] M. Goni and M. Valle, Phys. Rev. D 34, 648 (1986).

[80] J. J. Van der Bij, R. D. Pisarski, and S. Rao, Phys. Lett. B 179, 87 (1986).

[81] I. Vuorio, Phys. Lett. B 175, 176 (1986).

[82] I. Vuorio, Phys. Lett. B 181, 415 (1986).

[83] M. Kurkov and D. Vassilevich, J. High Energy Phys. 03 (2018) 072. 